\title{
Apatinib triggers autophagic and apoptotic cell death via VEGFR2/STAT3/PD-L1 and ROS/Nrf2/p62 signaling in lung cancer
}

Chunfeng Xie ${ }^{1 \dagger}$, Xu Zhou ${ }^{1+}$, Chunhua Liang ${ }^{1 \dagger}$, Xiaoting $\mathrm{Li}^{1+}$, Miaomiao Ge${ }^{1}$, Yue Chen ${ }^{1}$, Juan Yin ${ }^{2}$, Jianyun Zhu ${ }^{2^{*}}$ and Caiyun Zhong ${ }^{1,3^{*}}$

\begin{abstract}
Background: Recently, a variety of clinical trials have shown that apatinib, a small-molecule anti-angiogenic drug, exerts promising inhibitory effects on multiple solid tumors, including non-small cell lung cancer (NSCLC). However, the underlying molecular mechanism of apatinib on NSCLC remains unclear.

Methods: MTT, EdU, AO/EB staining, TUNEL staining, flow cytometry, colony formation assays were performed to investigate the effects of apatinib on cell proliferation, cell cycle distribution, apoptosis and cancer stem like properties. Wound healing and transwell assays were conducted to explore the role of apatinib on migration and invasion. The regulation of apatinib on VEGFR2/STAT3/PD-L1 and ROS/Nrf2/p62 signaling were detected. Furthermore, we collected conditioned medium (CM) from A549 and H1299 cells to stimulate phorbol myristate acetate (PMA)-activated THP-1 cells, and examined the effect of apatinib on PD-L1 expression in macrophages. The Jurkat T cells and NSCLC cells co-culture model was used to assess the effect of apatinib on T cells activation. Subcutaneous tumor formation models were established to evaluate the effects of apatinib in vivo. Histochemical, immunohistochemical staining and ELISA assay were used to examine the levels of signaling molecules in tumors.

Results: We showed that apatinib inhibited cell proliferation and promoted apoptosis in NSCLC cells in vitro. Apatinib induced cell cycle arrest at G1 phase and suppressed the expression of Cyclin D1 and CDK4. Moreover, apatinib upregulated Cleaved Caspase 3, Cleaved Caspase 9 and Bax, and downregulated BCl-2 in NSCLC cells. The colony formation ability and the number of CD133 positive cells were significantly decreased by apatinib, suggesting that apatinib inhibited the malignant and stem-like features of NSCLC cells. Mechanistically, apatinib inhibited PD-L1 and C-Myc expression by targeting VEGFR2/STAT3 signaling. Apatinib also inhibited PD-L1 expression in THP-1 derived macrophages stimulated by CM from NSCLC cells. Furthermore, apatinib pretreatment increased CD69 expression and IFN- $\gamma$ secretion in stimulated Jurkat T cells co-cultured with NSCLC cells. Apatinib also promoted ROS production and inhibited Nrf2 and p62 expression, leading to the autophagic and apoptotic cell death in NSCLC. Moreover, apatinib significantly inhibited tumor growth in vivo.
\end{abstract}

\footnotetext{
*Correspondence: njmuzhujy@njmu.edu.cn; cyzhong@njmu.edu.cn

${ }^{+}$Chunfeng Xie, Xu Zhou, Chunhua Liang and Xiaoting Li contributed equally to this work.

${ }^{2}$ Department of Laboratory, The Affiliated Suzhou Hospital of Nanjing

Medical University, Suzhou Municipal Hospital, Gusu School, Nanjing Medical University, 242 Guangji Rd, Suzhou 215008, China

${ }^{1}$ Department of Nutrition and Food Safety, School of Public Health, Nanjing Medical University, 101 Longmian Ave, Jiangning, Nanjing 211166, China

Full list of author information is available at the end of the article
} 4.0 International License, which permits use, sharing, adaptation, distribution and reproduction in any medium or format, as long as you give appropriate credit to the original author(s) and the source, provide a link to the Creative Commons licence, and indicate if changes were made. The images or other third party material in this article are included in the article's Creative Commons licence, unless indicated otherwise in a credit line to the material. If material is not included in the article's Creative Commons licence and your intended use is not permitted by statutory regulation or exceeds the permitted use, you will need to obtain permission directly from the copyright holder. To view a copy of this licence, visit http://creativecommons.org/ licenses/by/4.0/. The Creative Commons Public Domain Dedication waiver (http://creativecommons.org/publicdomain/zero/1. 0/) applies to the data made available in this article, unless otherwise stated in a credit line to the data. 
Conclusion: Our data indicated that apatinib induced autophagy and apoptosis in NSCLC via regulating VEGFR2/ STAT3/PD-L1 and ROS/Nrf2/p62 signaling.

\section{Highlights}

1. Apatinib suppressed proliferation, induced cell cycle arrest and apoptosis, and inhibited malignancy in NSCLC in vitro and in vivo.

2. Apatinib downregulated PD-L1 and C-Myc in NSCLC through VEGFR2/STAT3 pathway.

3. Apatinib inhibited PD-L1 expression in THP-1 derived macrophages stimulated by the conditioned medium from NSCLC cells and partially restored the activation of Jurkat T cells co-cultured with NSCLC cells.

4. Apatinib induced ROS generation and inhibited Nrf2 and p62 expression, leading to the autophagic and apoptotic cell death in NSCLC.

Keywords: NSCLC, Apatinib, Apoptosis, Autophagy, STAT3/PD-L1, ROS/Nrf2/p62

\section{Background}

Lung cancer is the leading cause of cancer-related deaths worldwide, with a very low 5-year survival rate (less than $15 \%)$ for all stage combined $[1,2]$. Non-small cell lung cancer (NSCLC) is the most common histologic subtype, accounting for approximately $85 \%$ of all lung cancer cases $[3,4]$. About half of the NSCLC patients present with an advanced stage at their first diagnosis, causing the subsequent therapy failure. Moreover, the outcome of locally advanced NSCLC patients remains poor, because of the almost inevitable metastasis, chemoresistance and subsequently tumor relapse $[5,6]$. Thus, alternatives to conventional treatment are urgently warranted for NSCLC.

NSCLC is a highly vascularized tumor and inhibition of angiogenesis has become a promising therapeutic approach [7]. It is reported that vascular endothelial growth factor (VEGF) plays an important role in the angiogenic signaling and vascular endothelial growth factor receptors (VEGFRs) are tyrosine kinases that serve as key regulators of this process. VEGFR2 is expressed by tumor cells and targeting this receptor may simultaneously affect tumor stroma (i.e. blood vessels) and tumor parenchyma, as it has been demonstrated in a variety of tumor types, including NSCLC [8]. Apatinib is a novel tyrosine kinase inhibitor (TKI) that approved and launched in China with promising therapeutic efficacy and tolerance in the treatment of multiple solid tumors [9]. Apatinib exerts its antigenic effects by inhibiting VEGFR-induced proliferation and migration of endothelial cells via highly and selectively targeting VEGFR2 [10]. Multiple clinical trials have demonstrated the antitumor activity of apatinib in monotherapy or combination therapy in advanced NSCLC patients [11-13].
Considering numerous molecules and signaling pathways influence the angiogenic response, in-depth understanding of the inhibition role of apatinib on NSCLC and its underlying mechanism are urgently needed for the further clinical usages.

Accumulating evidence have reported that antiangiogenic agents decrease the immunosuppression by modulating tumor microenvironment (TME) and exhibit synergetic effects when combined with immunotherapies [14]. Previous studies have shown that apatinib exerts its immunomodulatory activity in several tumor types. Zhao et al. reported that low-dose apatinib optimized the immunosuppressive TME and enhanced the immune response in murine lung carcinoma models and in patients with advanced nonsquamous NSCLC in a phase III trial [15]. Wang et al. showed that apatinib overcame the innate resistance to programmed death 1(PD-1)/programmed death ligand 1 (PD-L1) blockade and significantly improved the antitumoral activity of PD-1 antibody in advanced triple-negative breast cancer [16]. Further studies reported that apatinib inhibited PD-L1 expression by targeting signal transducer and activator of transcription 3 (STAT3) in osteosarcoma [17], while promoted its expression to enhance anti-PD-1 therapy in colon cancer [18]. In addition, apatinib improved the efficacy of immunotherapy by preventing the dysfunction of natural killer cells in hepatocellular carcinoma [19]. However, whether apatinib could affect PD-L1 and its regulatory mechanism in NSCLC remains unclear.

The nuclear factor erythroid-derived 2-like 2 (Nrf2) serves as a key transcriptional activator and its induction is of importance for protecting cells against oxidative and xenobiotic damage. Previous studies have demonstrated that Nrf2 disrupts oxidative stress mediated-cell 
death via scavenging cellular reactive oxygen species (ROS) and restoring redox balance. Moreover, abnormal activation of Nrf2 is closely associated with development and chemoresistance of a variety of human cancers, especially NSCLC [20]. Therefore, targeting Nrf2 and inducing ROS-mediated cell death might be a promising strategy in cancer treatment. Accumulating evidence have illustrated that Nrf2 also plays a vital role in autophagy regulation by forming a positive feedback with SQSTM1/p62, an autophagy adaptor protein. It is reported that insufficient autophagy causes p62 accumulation, which further sequesters kelch-like $\mathrm{ECH}$-associated protein 1 (Keap1), the negative regulator of $\mathrm{Nrf2}$, and leads to the stabilization of Nrf2 [21]. Thus, promoting autophagy and breaking the crosstalk between p62 and Nrf2 might be a potential option for cancer chemoprevention [22, 23]. Sun et al. showed that apatinib promoted ROS-dependent apoptosis and autophagy by negatively modulating Nrf2 and p62 in ovarian cancer cells [24]. Other studies also reported that apatinib inhibited cell growth and metastasis and promoted apoptosis by regulating autophagy in a variety of human cancers [25, 26]. However, whether apatinib could inhibit NSCLC by directly promoting autophagy and interfering with Nrf2 and p62 remains unclear.

Therefore, our present study aimed to explore the underlying mechanism of the inhibitory effects of apatinib on NSCLC. We showed that apatinib induced cell death of lung cancer by inhibiting cell proliferation, causing cell cycle arrest, and inducing apoptosis and autophagy. We also illustrated that apatinib suppressed malignant phenotype by inhibiting migration, invasion, and cancer stem cell-like properties. Mechanistically, apatinib decreased the expressions of PD-L1 and c-Myc by targeting VEGFR2/STAT3 pathway. In addition, apatinib induced autophagy and apoptosis by regulating ROS/Nrf2/p62 signaling. Hence, our study proposed the possible mechanism of apatinib against NSCLC and demonstrated the value of apatinib as a promising therapeutic drug for NSCLC.

\section{Materials and methods}

\section{Cell lines and cell culture}

Human NSCLC cell lines A549 and H1299, monocyte cell line THP-1 and Jurkat T cells were obtained from the Cell Bank of Chinese Academy of Sciences (Shanghai, China). The above cells were cultured in RPMI-1640 (Gibco, USA) with 10\% fetal bovine serum (FBS) and 1\% antibiotics.

To analyze the effect of apatinib on NSCLC, A549 and H1299 cells were treated with different concentrations of apatinib $(0,2,5,10 \mu \mathrm{M})$, using $0.1 \%$ dimethyl sulfoxide (DMSO) as the solvent control and cell morphology was observed under an inverted microscope ((Nikon,
Tokyo, Japan). For combination treatment, cells were exposed to apatinib with or without $5 \mathrm{mM}$ of N-acetyl-Lcysteine (NAC, Sigma-Aldrich, St. Louis, USA) or $20 \mathrm{ng} /$ mL of IL-6 (Peprotech, Rocky Hill, NJ, USA). Apatinb were obtained from Hengrui Medicine Co. Ltd. (Jiangsu, China) and dissolved in DMSO (Sigma-Aldrich).

\section{3-(4, 5-Dimethyl-2-thizolyl)-2, 5 diphenyltertazolium bromide (MTT) assay}

The effect of apatinib on cell viability was measured by MTT assay (Sigma-Aldrich). Briefly, A549 and H1299 cells (2000 cells/well) were cultured in 96-well plates and exposed to apatinib at indicated concentrations $(0$, $0.5,1,2,5,10,20,40 \mu \mathrm{M})$ for $24 \mathrm{~h}$ or $48 \mathrm{~h}$. After incubation with $20 \mu \mathrm{L}$ of MTT solution ( $5 \mathrm{mg} / \mathrm{ml}$ in PBS) for an additional $4 \mathrm{~h}$ at $37^{\circ} \mathrm{C}$, the supernatant solution was removed, $150 \mu \mathrm{L}$ of DMSO was added to dissolve the formazan crystal, and the absorbance of each well was measured with a microtiter reader (Tecan Infinite 200, Switzerland) at a wavelength of $490 \mathrm{~nm}$. The absorbance values for control cells were set as 1 for normalization.

\section{5-Ethynyl-2'-Deoxyuridine (EdU) assay}

The EdU assay was conducted using EdU Cell Proliferation Kit with Alexa Fluor488 (Beyotime, Shanghai, China) according to the manufacturer's protocol. Briefly, A549 and H1299 cells were plated onto the 12-well plates and incubated with apatinib $(0,2,5,10 \mu \mathrm{M})$ for $48 \mathrm{~h}$. After incubation of $10 \mu \mathrm{M}$ EdU for $2 \mathrm{~h}$, cells were fixed with $4 \%$ paraformaldehyde for $10 \mathrm{~min}$ and permeabilized with $0.3 \%$ Triton X-100 for $20 \mathrm{~min}$. Then, the incorporated EdU was visualized by means of a click reaction using Alexa Fluor 488 azide for $30 \mathrm{~min}$ and the nuclear DNA was stained with 4, 6-diamino-2-phenyl indole (DAPI, Beyotime) for $10 \mathrm{~min}$. Finally, the proliferative cells were observed using a fluorescence microscope (Nikon) and the percentage of EdU positive cells was assessed using the ImageJ software.

\section{Acridine orange/ethidium bromide (AO/EB) staining}

A549 and H1299 cells were cultured with apatinib for $24 \mathrm{~h}$, and the AO/EB staining was performed according the manufacturer's instructions (SenBeijia Biological Technology, Nanjing, China). Briefly, cells were incubated with $\mathrm{AO} / \mathrm{EB}$ solution in the dark for $15 \mathrm{~min}$ and the morphology of live and dead cells was observed immediately under a fluorescent microscope the percentage of dead cells was assessed using the ImageJ software.

Terminal deoxynucleotidyl transferase-mediated dUTP Nick end labeling (TUNEL) staining

The TUNEL assay was conducted using Colorimetric TUNEL Apoptosis Assay Kit (Beyotime, Shanghai, China) according to the manufacturer's protocol. Briefly, 
after incubation of apatinib for 48 h, A549 and H1299 cells were fixed, permeabilized, and then inactivated endogenous peroxidase by $0.3 \% \mathrm{H}_{2} \mathrm{O}_{2}$ for $20 \mathrm{~min}$. Subsequently, the cells were incubated with a mix solution containing enzyme terminal deoxynucleotide transferase (TdT) and biotinylated (Bio-16) dUTP in TdT buffer at $37^{\circ} \mathrm{C}$ for $60 \mathrm{~min}$ and the nuclei were stained with diaminobenzidine for $10 \mathrm{~min}$ and counterstained with hematoxylin. Finally, the apoptosis cells were visualized by an invert microscope.

\section{Flow cytometry analysis of cell cycle}

After treatment of apatinib for 48 h, A549 and H1299 cells were collected, resuspended, and fixed with precooled $70 \%$ ethanol at $-20^{\circ} \mathrm{C}$ overnight. After stained with the propidium iodide (PI) solution (BD, Biosciences, USA) for $15 \mathrm{~min}$, the distribution of cell cycle phase was measured by flow cytometry with a BD FACSArray (BD). A minimum of 20,000 cells were guaranteed before analysis.

\section{Plate colony formation assay}

A549 and H1299 cells were seeded into $60 \mathrm{~mm}$ diameter plates at a density of 100 cells/well. Cells were cultured with different concentrations of apatinib and the medium was renewed every 3 days. After 2 weeks' culturing, the colonies were fixed and dyed with crustal violet (Beyotime) for $30 \mathrm{~min}$. The colony images were recorded using a digital camera and the numbers of colonies (>50 cells/colony) were counted via Image J software.

\section{Anchorage-independent growth assay}

Soft agar dishes were precoated with $0.7 \%$ agarose (Beyotime) in RPMI-1640 medium. A549 and H1299 cells were plated at a density of 5000 cells/well in $0.35 \%$ agarose over the agar base and the medium was fleshed every 3 days with various concentrations of apatinib for 2 weeks, colonies with diameters $>50 \mu \mathrm{m}$ were counted.

\section{Flow cytometry analysis of $\mathrm{CD} 133$ positive cells}

The analysis was performed according to as previously described [27]. Briefly, A549 and H1299 cells were cultured in a stem cell specific serum-free medium and treated with apatinib for 7 days. The sphere-forming cells were collected and incubated with APC-conjugated human monoclonal CD133/1 (AC133) antibody at $4{ }^{\circ} \mathrm{C}$ for $15 \mathrm{~min}$. The percentage of $\mathrm{CD} 133$ positive cells was detected through flow cytometry (BD). Human monoclonal CD133/1 (AC133) antibody was purchased from Miltenyi Biotech (Teterow, Germany).

\section{Wound healing assay}

A549 and H1299 cells were seeded in 6-well plates for adherent culture and a sterile pipette tip was used to make the scratches. The wounded cells were then treated with apatinib $(0,2,5,10 \mu \mathrm{M})$ after removing debris by PBS for $48 \mathrm{~h}$. The scratch area was captured at $0 \mathrm{~h}$ and $48 \mathrm{~h}$ and the migration rate were measured by Image J.

\section{Transwell assays}

A549 and H1299 cells were treated with apatinib for 48 $\mathrm{h}$ and were resuspended at a density of $5 \times 10^{4}$ cells/ well $(200 \mu \mathrm{L})$ and seeded in the upper chambers (24-well migration chambers, $8.0 \mu \mathrm{m}$ pore membrane, Corning, New York, USA) in the serum-free medium. The upper chambers were precoated with Matrigel (Corning) for cell invasion, not for cell migration. Then, the lower chambers were filled with $800 \mu \mathrm{L}$ of the medium with $10 \%$ FBS. After $24 \mathrm{~h}$ incubation, the non-migrated and non-invaded cells in the upper chambers were removed by cotton swabs, and the migrated or invaded cells on the undersurface of the chambers were fixed and stained with crystal violet. The images were observed using a microscope and migrated and invaded cells were measured by Image $\mathrm{J}$.

\section{ROS detection}

A549 and H1299 cells $\left(1 \times 10^{6}\right.$ cells/well $)$ were seeded in 6 -well plates. After treatment with apatinib for $24 \mathrm{~h}$, cells were incubated with $5 \mathrm{mM}$ of $2^{\prime}, 7^{\prime}$-dichlorodihydrofluorescein diacetate (DCFDA, Sigma-Aldrich) and placed in a shaker at $37^{\circ} \mathrm{C}$ for $20 \mathrm{~min}$. The DCF fluorescence intensity was then immediately detected using a fluorescence microscopy.

\section{Conditioned medium preparation and macrophages treatment}

To obtain conditioned medium (CM) of A549 and H1299 cells, both cells, up to $80 \%$ confluence, were washed and cultured with fresh medium for additional $24 \mathrm{~h}$. The cell-medium was collected and centrifuged at $3000 \mathrm{rpm}$ for $10 \mathrm{~min}$ at $4{ }^{\circ} \mathrm{C}$.

To analyze the effects of apatinib on macrophages, THP-1 cells were stimulated by pretreatment with 100 $\mathrm{ng} / \mathrm{mL}$ phorbolmyristate acetate (PMA, Sigma-Aldrich) for $48 \mathrm{~h}$. The THP-1 derived macrophages were then washed and cultured with fresh medium or CM from A549 or H1299 cells with or without $10 \mu \mathrm{M}$ apatinib for another $4 \mathrm{~h}$.

\section{Co-culture assay}

A549 and H1299 cells were seeded onto 24-well plate at a density of $5 \times 10^{4}$ and treated with or without $10 \mu \mathrm{M}$ apatinib for $24 \mathrm{~h}$, then the culture medium was aspirated 
and replaced with fresh 1640 medium containing 10\% FBS. The Jurkat $\mathrm{T}$ cells were pre-activated with $2 \mu \mathrm{g} / \mathrm{ml}$ soluble anti-CD3 (eBioscience, San Diego, CA, USA) and $1 \mu \mathrm{g} / \mathrm{ml}$ soluble anti-CD28 (eBioscience) for $24 \mathrm{~h}$, and were then mixed with A549 and H1299 control cells or apatinib pretreated cells at a density of $5 \times 10^{5}$ for coculture. After $24 \mathrm{~h}$, the culture medium was collected for IFN- $\gamma$ ELISA assay and Jurkat cells were collected for flow cytometry analysis.

\section{Flow cytometry analysis of CD69 expression}

Jurkat $\mathrm{T}$ cells from the co-culture system were harvested and washed with PBS. After staining with the PE-cy7 conjugated anti-CD69 antibody (Biolegend, San Diego, CA, USA) in PBS for $30 \mathrm{~min}$ in the dark, the stained cells were acquired by flow cytometry analysis. Mean fluorescent intensity was calculated.

\section{Enzyme-linked immunosorbent assay (ELISA) for interferon- $\gamma$ (IFN- $\gamma$ )}

IFN- $\gamma$ level in medium of the co-culture system was determined using Human IFN- $\gamma$ ELISA kit (CUSABIO, Wuhan, China) according to the manufacturer's instructions. Optical density was measured at $450 \mathrm{~nm}$, and the amount of IFN- $\gamma$ was calculated from a standard curve prepared using the recombinant protein.

\section{Quantitative real-time PCR (qRT-PCR) analysis}

Total RNA was isolated with TRIzol reagent (Invitrogen, Carlsbad, CA, USA) according to the manufacturer's protocol and cDNA was completed using a cDNA synthesis kit (Applied Biological Materials, Canada). qRTPCR were performed using the EvaGreen $2 \times$ qPCR MasterMix (Applied Biological Materials) and detected by a LightCycler96 real-time detection (Roche, Basel, Switzerland). The PCR primer sequences were as follows: VEGFR2: forward: 5'-TGGTCAGGCAGCTC ACAGTCC-3', reverse: 5'-GTTCCGGTTCCCATCCTT CAATAC-3', PD-L1: forward: 5'-CATGTCAGGC TGAGGGCTAC-3', reverse: 5' -TGGAATTGGTGGTG GTGGTC-3'; GAPDH: forward: 5'-CAAGGTCACC ATGACAACTTTG-3', reverse: 5'-GTCCACCACCCT GTTGCTGTAG-3'. GAPDH was regarded as an internal reference. The relative expression levels were calculated by the $2^{-\Delta \Delta C t}$ method. The PCR primers were synthesized by Tsingke Biological Technology (Beijing, China).

\section{Western blot analysis}

Total proteins were extracted from cells and tumor tissues using RIPA buffer supplemented with $1 \%$ protease inhibitors on ice and quantified by BCA protein assay kit (Beyotime). Equal amounts of protein samples were separated by SDS-polyacrylamide gel electrophoresis, transferred onto polyvinylidene fluoride membranes (Millipore, Billerica, MA, USA), blocked with 5\% non-fat dried milk, and then incubated with the primary antibody overnight at $4{ }^{\circ} \mathrm{C}$. The membranes were incubated with horseradish peroxidase (HRP)-conjugated secondary antibodies for $1 \mathrm{~h}$ and detected using an enhanced chemiluminescence kit (Millipore, Billerica, MA, USA). GAPDH was used as the loading control.

The primary antibodies to CDK4 (cat. no. 11026-1AP), Cyclin D1 (cat. no. 26939-1-AP), Cleaved Caspase 9 (cat. no. 10380-1-AP), Cleaved Caspase 3 (cat. no. 19677-1-AP), Bcl-2 (cat. no. 12789-1-AP), Bax (cat. no. 50599-2-lg), E-cadherin (cat. no. 20874-1-AP), Vimentin (cat. no. 10366-1-AP), MMP2 (cat. no. 10373-2-AP), Nrf2 (cat. no. 16396-1-AP), LC3 (cat. no. 14600-1-AP), p62 (cat. no. 18420-1-AP), Beclin 1 (cat. no. 11306-1AP), ATG5 (cat. no. 10181-2-AP), and c-Myc (cat. no. 10828-1-AP) were purchased from Proteintech (Rosemont, IL, USA). VEGFR2 (cat. no. AF6281), phosphorylated-VEGFR2 (p-VEGFR2, Tyr1175, cat. no. AF4426), phosphorylated-STAT3 (p-STAT3, Tyr705, cat. no. AF3293), and STAT3 (cat. no. AF6293) were purchased from Affinity (Cincinnati, USA). PD-L1 (cat. no. 13684) was purchased from Cell Signaling Technology (Boston, MA, USA). GAPDH (cat. no. AP0063) was purchased from Bioworld (Nanjing, China).

\section{Immunofluorescence staining assay}

A549 and H1299 cells $\left(1 \times 10^{5}\right.$ cells/well $)$ were plated onto 12-well plates and exposed to apatinib for $48 \mathrm{~h}$. The cells were fixed, permeabilized, blocked with goat serum, and then incubated with antibody against Cleaved Caspase 3 (1:200 dilution, Proteintech), Ecadherin (1:400 dilution, Proteintech), Vimentin (1:400 dilution, Proteintech), p-STAT3 (1:200 dilution, Affinity), Nrf2 (1:200 dilution, Proteintech), p62 (1:200 dilution, Proteintech), and LC3 (1:200 dilution, Proteintech) overnight at $4{ }^{\circ} \mathrm{C}$. Followed by incubation with FITCconjugated secondary antibody for $45 \mathrm{~min}$, the nuclei were stained with DAPI for $10 \mathrm{~min}$. Finally, the fluorescence images were obtained by a confocal microscopy.

\section{Tumor xenografts}

A $100 \mu \mathrm{L}$ suspension of $5 \times 10^{6}$ A549 cells were injected subcutaneously into the right axilla of nude mice (SPF grade, 4-5 weeks old, male). Fourteen days after injection, the mice were randomly divided into the control group and apatinib treatment group $(n=5$ for each group). Apatinib was initially dissolved in DMSO as the stock solution, and was then diluted with saline and the final concentration of DMSO in the oral gavage solution was $2 \%$. The mice in the treatment group were administrated daily with apatinib at $100 \mathrm{mg} / \mathrm{kg}$ body weight by oral gavage, while the mice in the control group were 
administrated with saline solution with equal concentration of DMSO (2\%). The body weight of each mouse was measured every 3 day after injection. At the termination of the experiment, the mice were sacrificed and the tumors were removed. The weight, width and length of each tumor was recorded and the tumor volume was calculated using a formula: [width ${ }^{2} \times$ length] $/ 2$. Subsequently, tumor tissues were frozen for western blot analysis or embedded for histochemical and immunohistochemistry evaluation.

$\mathrm{BALB} / \mathrm{c}$ nude mice were purchased from the Animal Research Center of Nanjing Medical University. Animals' care was performed according to the institutional guidelines, and the animal protocol was approved by the Animal Care and Welfare Committee of Nanjing Medical University (IACUC-1907001).

\section{Measurement of VEGFR2}

Total proteins from mice tumor tissues were lysed using RIPA buffer. The VEGFR2 expression level in tumor tissues was quantified using a mouse Enzyme-Linked Immunosorbent Assay (ELISA) kit followed by the manufacturer's instructions (Abcam, Cambridge, MA, USA). Optical density was measured at $450 \mathrm{~nm}$, and the amount of VEGFR2 was calculated from a standard curve prepared using the recombinant protein.

\section{Histochemical or immunohistochemical staining}

The tumor tissues were fixed with $4 \%$ paraformaldehyde, dehydrated in a series of graded ethanol solutions, and embedded in paraffin for slicing into $5 \mu \mathrm{m}$ slides. Afterwards, the slides were used for subsequent hematoxylineosin (H\&E) or immunohistochemical staining. The antibodies used for immunohistochemistry were mouse anti-Cyclin D1 (1:100 dilution, Proteintech), rabbit antiCleaved Caspase 3 (1:100 dilution, Proteintech), rabbit anti-E-cadherin (1:100 dilution, Proteintech), rabbit antiVimentin (1:100 dilution, Proteintech), rabbit anti-Nrf2 (1:100 dilution, Proteintech), rabbit anti-p62 (1:100 dilution, Proteintech), rabbit anti-LC3 (1:100 dilution, Proteintech), rabbit anti-VEGFR2 (1:100 dilution, Affinity), rabbit anti-p-STAT3 (1:100 dilution, Proteintech), rabbit anti-c-Myc (1:100 dilution, Proteintech), and rabbit antiPD-L1 (1:100 dilution, Cell Signaling Technology). Eventually, the images were obtained under an optical microscope (Nikon).

\section{Statistical analysis}

Statistical analysis was carried out using GraphPad Prism (Inc., San Diego, CA, USA). The data were presented as mean \pm SD. Statistical analysis between two groups were compared using unpaired two-tailed Student's $t$ test. Statistics analysis among multiple groups were compared using one-way analysis of variance (ANOVA). A $P$ value $<0.05$ was considered as significant difference.

\section{Results \\ Apatinib suppressed cell proliferation in NSCLC cells}

To explore whether apatinib impacted the cell viability and proliferation of NSCLC, A549 and H1299 cells were treated with various concentrations of apatinib for $24 \mathrm{~h}$ or $48 \mathrm{~h}$. MTT assay showed that apatinib inhibited cell viability of lung cancer cells in a concertation- and timedependent manner (Fig. 1a). We observed that apatinib at $2 \mu \mathrm{M}$ already induced significant inhibition of cell viability in both cells for $48 \mathrm{~h}$ treatment, thus, we selected apatinib at concentrations of $2,5,10 \mu \mathrm{M}$ for our further experiments. After $48 \mathrm{~h}$ treatment of apatinib, we noticed that A549 and H1299 cells showed round and shrunk morphology and the morphological changes became more obvious with the increasing concentration of apatinib (Fig. 1b). Consistently, apatinib induced a markedly decrease in the percentage of EdU-positive proliferative cells (Fig. 1c). Furthermore, AO/EB staining showed that the death cell numbers were significantly increased in apatinib-treated A549 and H1299 cells (Fig. 1d). Together, these results suggested that apatinib inhibited the growth of NSCLC cells.

\section{Apatinib arrested cells at $\mathrm{G} 1$ phase and induced apoptosis in NSCLC cells}

To further investigate the mechanism of apatinibinduced suppression of proliferation in NSCLC cells, cell cycle distributions of A549 and H1299 cells were determined by flow cytometry after apatinib treatment. Our results showed that apatinib at $5 \mu \mathrm{M}$ and $10 \mu \mathrm{M}$ blocked the G1 to $\mathrm{S}$ cell cycle transition in both cells (Fig. 2a and b). We further analyzed the impact of apatinib on cell cycle related factors. As shown in Fig. 2c, apatinib concentration-dependently downregulated the expressions of G1 phase-regulated proteins CDK4 and Cyclin D1 in A549 and H1299 cells.

Since apoptosis has been thought to be a major antiproliferative mechanism of anticancer drug, we next examined the role of apatinib on apoptosis in NSCLC cells. TUNEL staining assay showed that the rate of apoptotic cells was significantly increased in A549 and H1299 cells upon apatinib treatment (Fig. 2d). Western blot analysis showed that apatinib upregulated the levels of apoptosis proteins Cleaved Caspase 9, Cleaved Caspase 3, and Bax, but downregulated the level of antiapoptosis protein $\mathrm{Bcl}-2$ (Fig. 2e). Furthermore, immunofluorescence staining analysis also showed the protein expression of Cleaved Caspase 3 was significantly increased in apatinib treated-lung cancer cells (Fig. 2f). Taken together, these results suggested that apatinib 


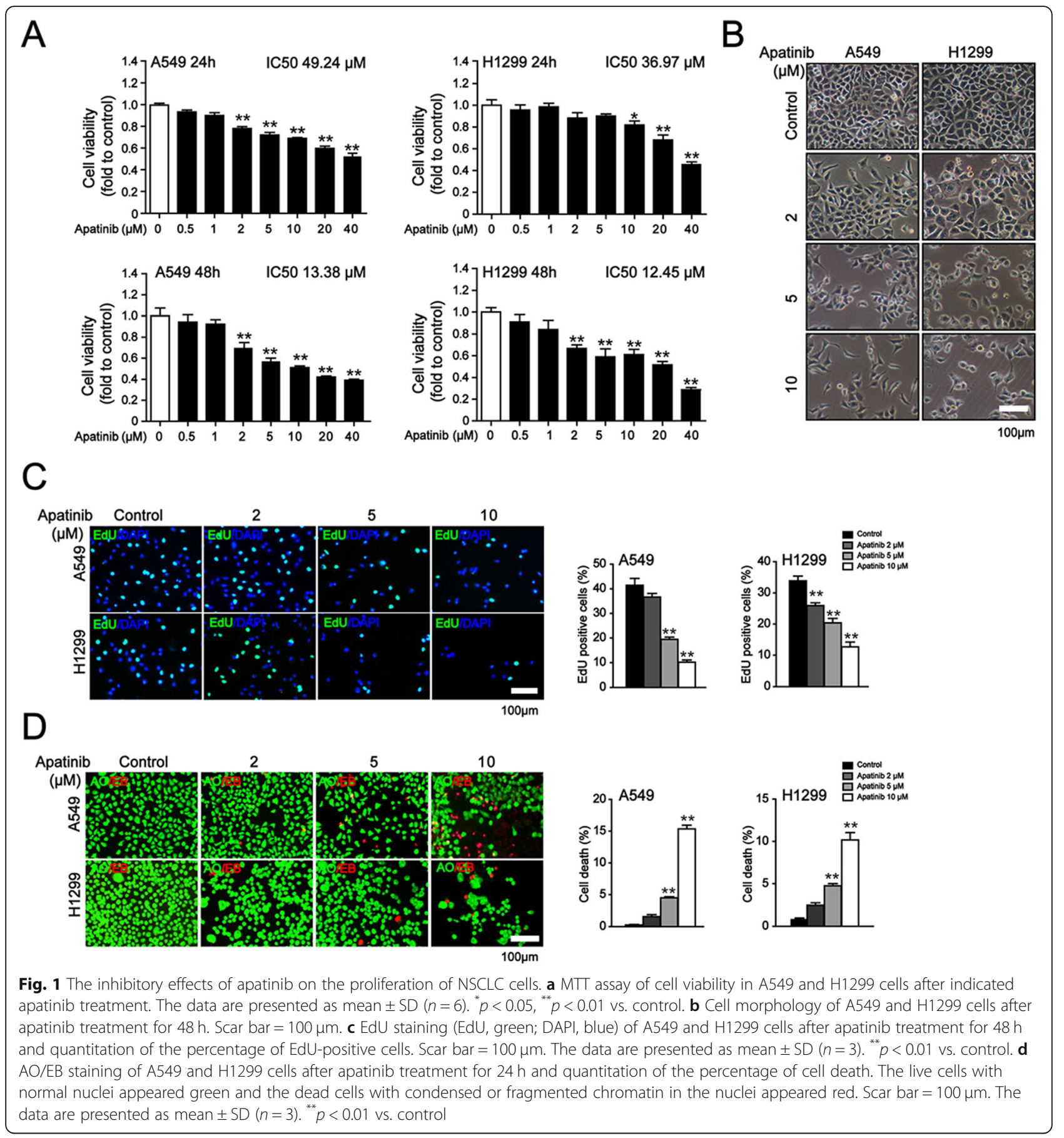

induced G1 phase cell cycle arrest and apoptosis in NSCLC cells.

\section{Apatinib inhibited the malignant and stemness phenotype of NSCLC cells}

To analyze the role of apatinb on the malignant phenotype of NSCLC, plate and soft agar colony formation assays were performed. We found that the plate colony formation ability of both A549 and H1299 cells was decreased in a concentration-dependent manner after apatinib treatment (Fig. 3a). Meanwhile, soft agar colony formation assay also showed a similar inhibition of apatinib on anchorageindependent growth capability of NSCLC cells (Fig. 3b).

The cancer stem cell hypothesis posits that the existence of cancer stem cells is a vital mechanism for cancer initiation, development, and chemoresistance. CD133 is recognized as a specific surface marker of lung cancer stem cells and CD133-positive cells exhibit stem cell-like properties 


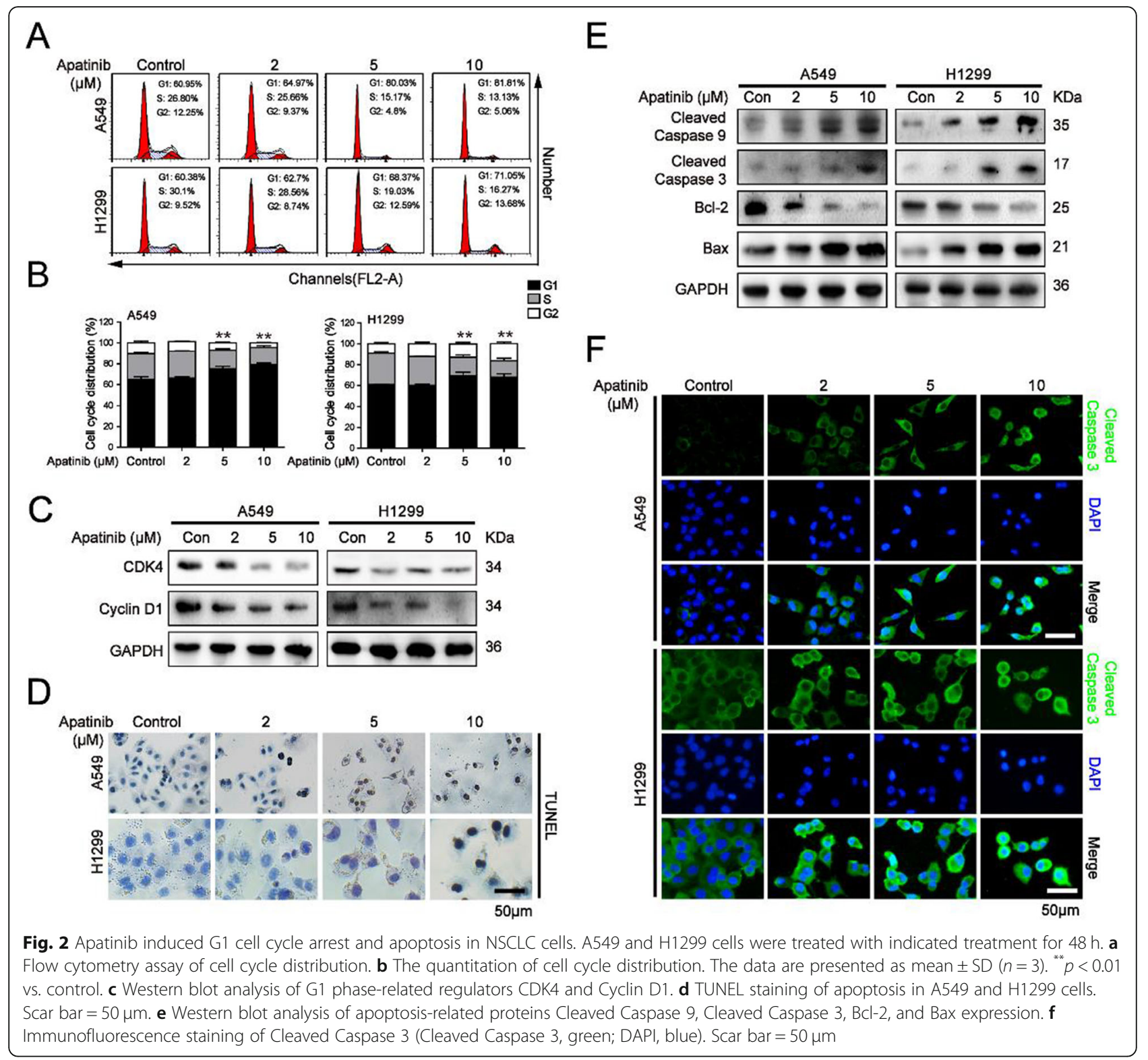

$[28,29]$. We further investigated the effect of apatinib on the stemness phenotype of NSCLC cells. A549 and H1299 cells were cultured with indicated concentrations of apatinib in a stem cell specific serum-free medium for 7 days and the numbers of CD133-positive cells were detected by flow cytometry assay. As shown in Fig. 3b, the percentage of CD133-positive cells was significantly downregulated in apatinib treated-cells. These results suggested that apatinib inhibited malignant growth and cancer stem cell-like properties in NSCLC cells.

\section{Apatinib suppressed the migration and invasion capacities of in NSCLC cells}

To assess the influence of apatinib on the metastatic potency of NSCLC cells, wound healing and transwell assays were carried out. As shown in Fig. 4a and b, apatinib retarded wound closure in a concertation-dependent manner. Transwell assay further showed that after $48 \mathrm{~h}$ treatment of apatinib, the numbers of migrated cells were significantly reduced when compared to that of the control cells. In addition, the numbers of A549 and H1299 cells that invaded onto the lower chamber through extracellular matrix gels were also markedly decreased following apatinib treatment (Fig. 4c-e). These results indicated that apatinib effectively suppressed the migration and invasion capacity of NSCLC cells.

Given the epithelial-mesenchymal transition (EMT) is the initiating step towards invasion and distant metastasis, we next investigated the expression of EMT-related markers upon apatinib treatment. Immunofluorescence 


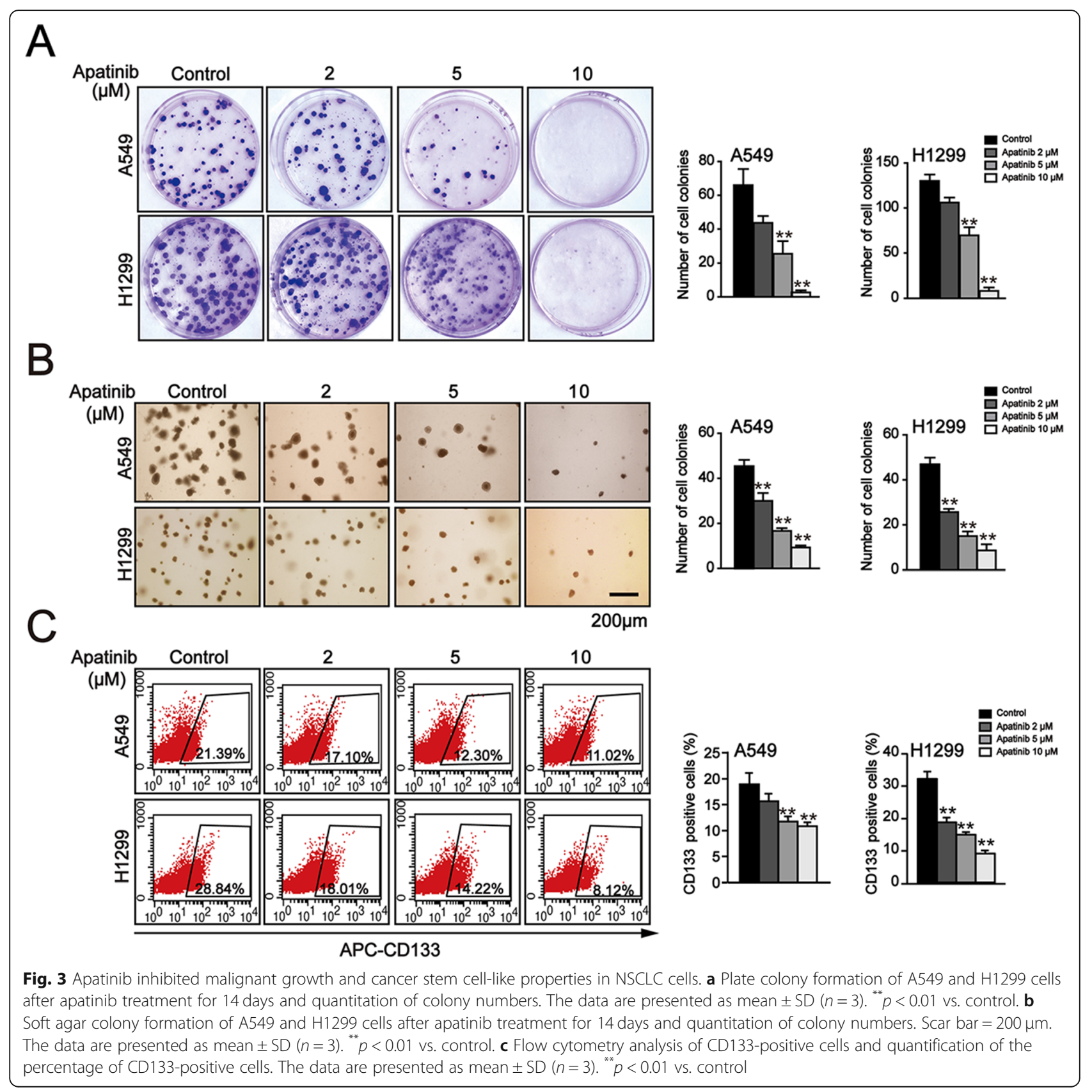

staining analysis showed that apatinib upregulated the expression of epithelial-related marker E-cadherin and concomitantly downregulated the expression of mesenchymal-related marker Vimentin in A549 and H1299 cells (Fig. 4f). Moreover, Western blotting further confirmed that the protein level of E-cadherin was increased, whereas the levels of Vimentin and MMP2 were decreased in apatinib treated-cells (Fig. 4g). Taken together, these results indicated that apatinib inhibited metastasis by repressing the EMT process in NSCLC cells.
Apatinib downregulated VEGFR2/STAT3/PD-L1 signaling in NSCLC cells and reduced the immunosuppressive TME It is acknowledged that apatinib highly selectively targets VEGFR2 and blocks VEGF-induced proliferation and migration of endothelial cells. Previous studies have demonstrated that apatinib exerts antitumor abilities in multiple solid tumors by targeting VEGFR2/STAT3 pathway. Accordingly, we explored whether apatinib inhibited NSCLC cells by regulating VEGFR2/STAT3 signaling pathway. As shown in Fig. 5a, after $48 \mathrm{~h}$ treatment, apatinib significantly downregulated the protein 


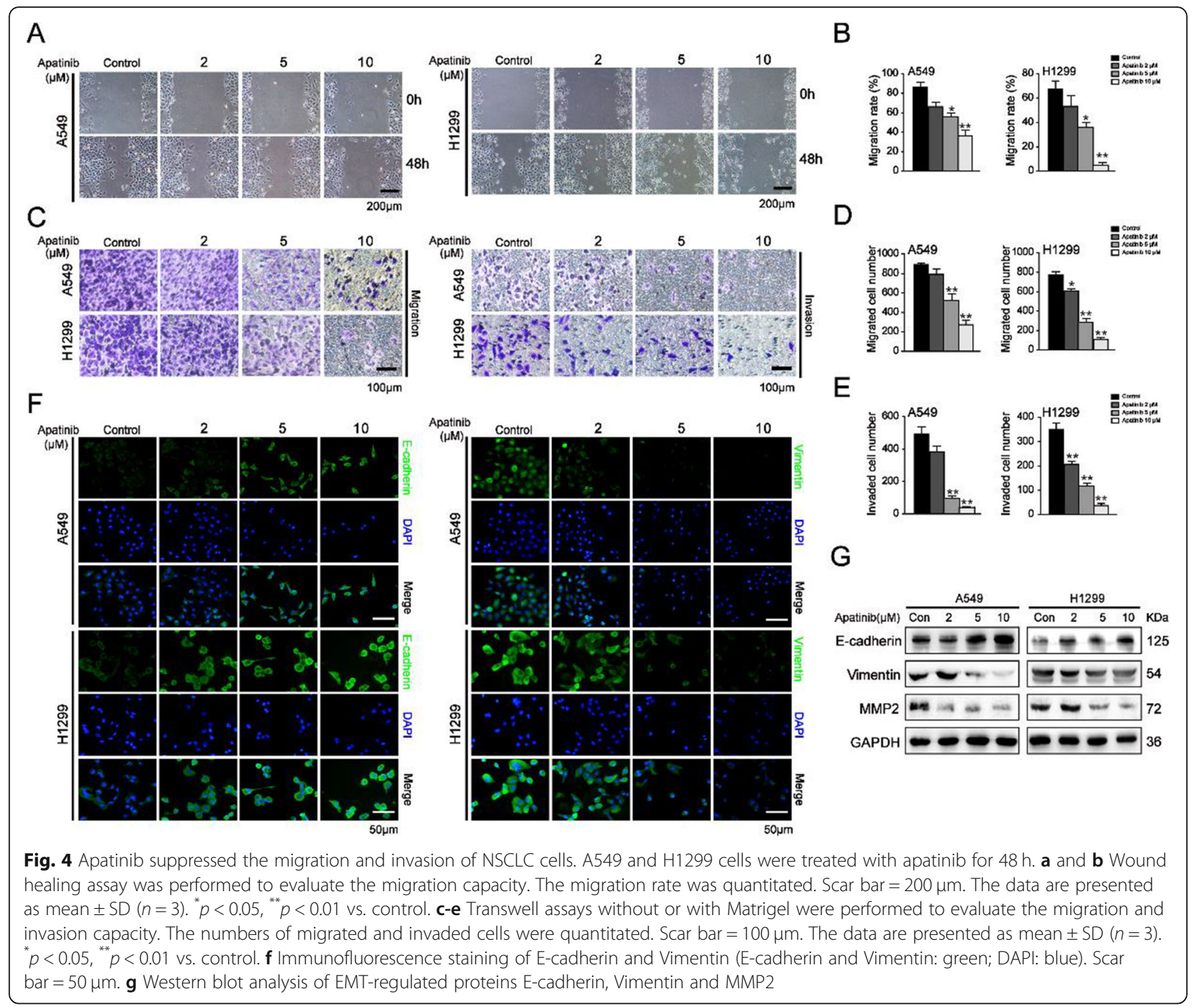

and mRNA expression of VEGFR2 and the levels of pVEGFR2 (Tyr1175) in A549 and H1299 cells. Moreover, our results showed that the protein level of p-STAT3 was time- and concentration-dependently decreased in apatinib-treated cells in comparison with that in the control cells (Fig. 5b and c). Immunofluorescence staining assay also showed that apatinib markedly suppressed p-STAT3 expression (Fig. 5d). Additionally, the protein levels of c-Myc and PD-L1, the major downstream targets of STAT3 signaling displayed a consistent decline upon apatinib treatment (Fig. $5 \mathrm{~b}$ and c). To further analyze whether apatinib inhibited NSCLC cells via STAT3 signaling, we treated A549 and H1299 cells with apatinb after pretreatment of $20 \mathrm{ng} / \mathrm{mL}$ interleukin-6 (IL-6), a major mediator of inflammation and activator of STAT3. The results showed that IL-6 induced the activation of STAT3 pathway, while the expression levels of IL-6 upregulated-p-STAT3, c-Myc, and PD-L1 were reversed by apatinib (Fig. 5e).
To further explore whether apatinib could impact PDL1 expression in tumor-associated macrophages, we treated THP-1 derived macrophages with $10 \mu \mathrm{M}$ apatinib for $4 \mathrm{~h}$ after stimulation of CM from A549 and H1299 cells. Western blot and qRT-PCR analysis showed that apatinib markedly inhibited the protein and mRNA level of PD-L1 in CM-treated macrophages, but had no obvious impact on PD-L1 expression in macrophages without CM stimulation (Fig. 5f). The results indicated that apatinib inhibited tumor-associated macrophages in TME.

We further examined whether apatinib downregulated PD-L1 in NSCLC and THP-1 cells was associated with reduced immunosuppressive activity of these cells against T-cells. Thus, we established Jurkat T cells and A549 or H1299 cells co-culture model. As CD69 is the earliest cell surface marker of activated $\mathrm{T}$ cells [30], we then examined the effect of apatinib on the expression of CD69 in activated 


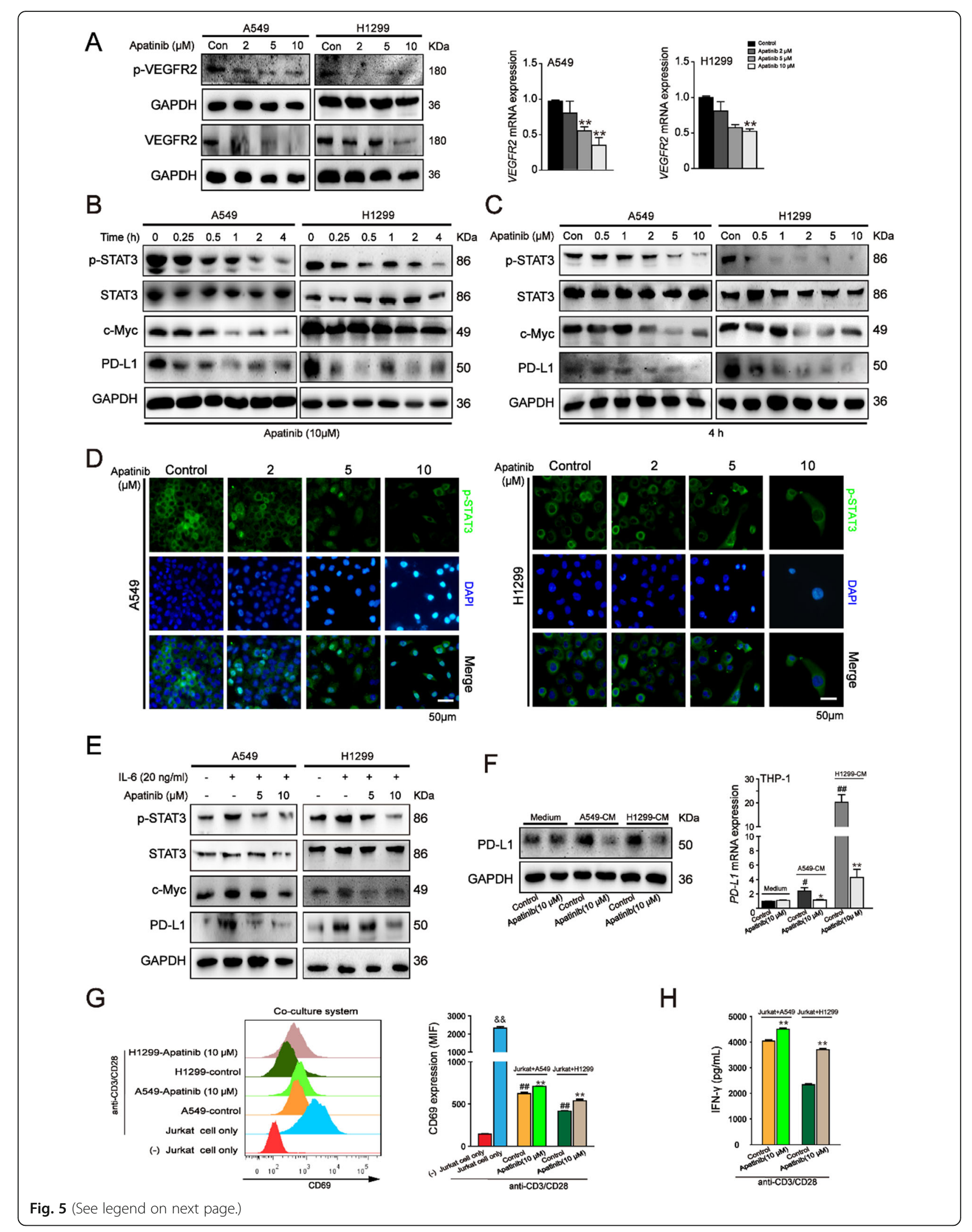


(See figure on previous page.)

Fig. 5 Apatinib downregulated VEGFR2/STAT3/PD-L1 pathway in NSCLC cells and reduced the immunosuppressive TME. a Western blot analysis of VEGFR2 and p-VEGFR2 (Tyr1 175) expression and qRT-PCR analysis of VEGFR2 expression in A549 and H1299 cells after aptinib treatment for 48 h. The data are presented as mean \pm SD $(n=3) .{ }^{* *} p<0.01$ vs. control. $\mathbf{b}$ and $\mathbf{c}$ Western blot analysis of p-STAT3, STAT3, c-Myc and PD-L1 expressions in A549 and H1299 cells after indicated treatment. d Immunofluorescence staining of p-STAT3 in A549 and H1299 cells after apatinib treatment for $4 \mathrm{~h}$ ( $\mathrm{p}$-STAT3: green; DAPI: blue). Scar bar $=50 \mu \mathrm{m}$. e Western blot analysis of p-STAT3, STAT3 and PD-L1 expressions in A549 and H1299 cells after apatinib treatment with or without pretreatment of IL-6 for $48 \mathrm{~h}$. f Western blot and qRT-PCR analysis of PD-L1 expression in THP-1-derived macrophages with or without stimulation with CM from A549 and H1299 cells. The data are presented as mean \pm SD $(n=3)$. ${ }^{*} p<$ $0.05,{ }^{* *} p<0.01$ vs. A549 or H1299-CM control. ${ }^{*} p<0.05,{ }^{*} p<0.01$ vs. medium control. $\mathbf{g}$ and $\mathbf{h}$ Jurkat cells were activated by stimulation with anti CD3/CD28 antibodies and then co-cultured with non-treated or apatinib $(10 \mu \mathrm{M})$ pretreated A549 or H1299 cells; the Jurkat cells were collected for CD69 detection by flow cytometry $(\mathbf{g})$ and the co-culture medium was collected for IFN- $\gamma$ secretion by ELISA assay (h). The data are presented as mean \pm SD $(n=3)$. ${ }^{\text {\& } ~} p<0.01$ vs $(-)$ Jurkat cell only group. ${ }^{* *} p<0.01$ vs. A549+ Jurkat or H1299 + Jurkat control group. ${ }^{\# \#} p<0.01$ vs. Jurkat cells only stimulated with anti CD3/CD28 antibodies group

Jurkat $\mathrm{T}$ cells. As shown in Fig. 5g, Jurkat T cells exhibited elevated CD69 level after stimulation with anti CD3/CD28 antibodies, and co-culture of activated Jurkat T cells with A549 or H1299 cells significantly decreased its expression. As expected, the level of CD69 was upregulated in activated Jurkat $\mathrm{T}$ cells when cocultured with apatinib $(10 \mu \mathrm{M})$ pretreated NSCLC cells when compared with non-treated control cells (Fig. 5g). We also examined the secretion of IFN- $\gamma$, which was produced by the effector T cells in the coculture cell medium. Our results showed that pretreatment of apatinib increased IFN- $\gamma$ production in activated Jurkat $\mathrm{T}$ cells in comparison with nontreated control cells (Fig. 5h). Taken together, our results suggested that apatinib partially restored the activation of Jurkat $\mathrm{T}$ cells and reduced the immunosuppressive TME.

\section{Apatinib induced autophagic and apoptotic cell death via ROS/Nrf2/p62 pathway in NSCLC cells}

Previous studies demonstrated that anticancer drugs triggered apoptosis of tumor cells by inducing the excessive generation of ROS. We examined the role of apatinib on ROS production in NSCLC cells. As shown in Fig. 6a, after $24 \mathrm{~h}$ treatment of apatinib, the production of ROS in A549 and H1299 cells were significantly increased. Since the antioxidant system determines the cellular level of ROS, we further evaluated the expression of the antioxidant enzyme Nrf2 after apatinib treatment. We observed that short time $(0.25 \mathrm{~h}$ and $0.5 \mathrm{~h})$ treatment of $10 \mu \mathrm{M}$ apatinib did not significantly inhibit Nrf2 expression in A549 cells, whereas Nrf2 expression was increased in apatinib-treated $\mathrm{H} 1299$ cells for $0.5 \mathrm{~h}$. With the prolong of apatinib treatment, the protein level of Nrf2 was eventually decreased in both cells (Fig. 6b). Western blot and immunofluorescence staining assays further showed that Nrf2 expression was decreased in a concentration-dependent manner in apatinib treatedcells (Fig. $6 \mathrm{c}$ and d). In addition, the inhibition of apatinib on Nrf2 expression was obviously reversed by NAC, a ROS scavenger (Fig. 6g). These results suggested that apatinib inhibited NSCLC by inducing ROS production and disrupting antioxidant defense system.

We next explored whether apatinib-induced ROS production caused autophagy in NSCLC. It is reported that in the process of autophagy, the cytosolic LC3-I is converted to autophagic vesicle-associated LC3-II. Our results showed that the expression level of LC3-II form was markedly upregulated after apatinib treatment (Fig. 6b, c and f). Additionally, an increase in the expressions of autophagy related-proteins Beclin1 and ATG5 and a decrease in p62 expression were significantly detected in apatinib treated-A549 and H1299 cells (Fig. 6c and e). Furthermore, our results showed that NAC treatment obviously attenuated apatinib-induced autophagy and apoptosis. As shown in Fig. 6g, apatinib downregulatedNrf2, p62, and Cyclin D1 and upregulated-LC3-II and Cleaved Caspase 3 were partially reversed by NAC. Collectively, these results suggested that apatinib induced autophagic and apoptotic cell death through ROS/Nrf2/ p62 pathway.

\section{Apatinib inhibited the growth of NSCLC cells in vivo}

To investigate the effects of apatinib on tumor growth in vivo, A549 cells were inoculated into BALB/c nude mice by subcutaneous injection Fourteen days after injection, the mice were randomly divided into the control group and apatinib treatment group. The mice in the treatment group were orally administrated daily with apatinib at $100 \mathrm{mg} / \mathrm{kg}$ body weight, while the mice in the control group were administrated with vehicle solution. After administration for another 12 days, both tumor volume and tumor weight were significantly reduced in apatinib-treated mice (Fig. 7a-c), indicating the inhibitory effects of apatinib on tumor growth in vivo. Meanwhile, we also measured the body weight of each mouse during the experiment. We found that there were no obvious changes in the body weight of mice after apatinib administration, suggesting that the tolerance and efficiency of apatinib treatment (Fig. 7d).

Moreover, we showed that apatinib inhibited proliferation and induced apoptotic and autophagic death in lung 


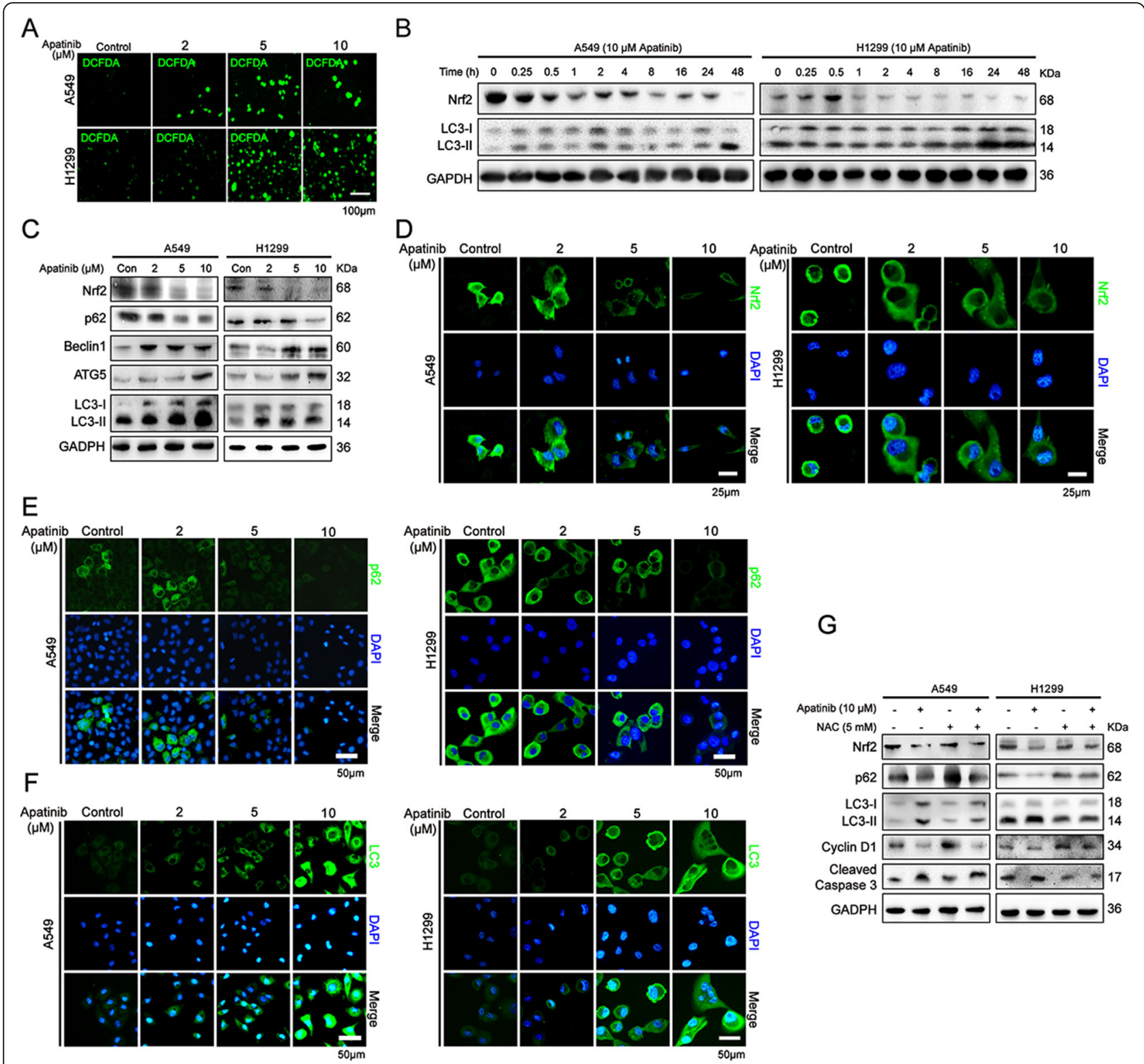

Fig. 6 Apatinib induced autophagic and apoptotic cell death via ROS/Nrf2/p62 pathway in NSCLC cells. a The representative images of fluorescence intensity of DCFH-DA in apatinib-treated A549 and H1299 cells. Scale bar $=100 \mu \mathrm{m}$. b Western blot analysis of Nrf2 and LC3 expressions in apatinib (10 MM)-treated A549 and H1299 cells for 0-48 h. c Western blot analysis of Nrf2, p62, Beclin1, ATG5, and LC3 expressions in A549 and H1299 cells after various concentrations of apatinib treatment for 48 h. d-f Immunofluorescence staining of Nrf2 (d), p62 (e), and LC3 (f) in A549 and H1299 cells after indicated treatment (Nrf2, p62, and LC3: green; DAPI: blue). Scar bar = 50 m. g Western blot analysis of Nrf2, p62, LC3, Cyclin D1 and Cleaved Caspase 3 expressions in A549 and H1299 cells after apatinib with or without NAC treatment for $48 \mathrm{~h}$

cancer tumors, as evidenced by the decreased expression of Cyclin D1, Nrf2 and p62, but the increased expression of Cleaved Caspase 3 and LC3-II (Fig. 7f-i). Immunohistochemical staining analysis also showed that apatinib upregulated the level of E-cadherin and downregulated the level of Vimentin (Fig. 7j). ELISA and immunohistochemical staining analysis confirmed that apatinib markedly reduced VEGFR2 level in tumors (Fig. 7e and j). Immunohistochemical staining results further showed that the protein levels of p-STAT3, c-Myc, and PD-L1 were also downregulated in apatinib-treated tumors (Fig. $7 j)$. Taken together, consistent with in vitro studies, our results suggested that apatinib inhibited the tumor growth of NSCLC cells by targeting both Nrf2 and VEGFR2/STAT3 signaling in vivo.

\section{Discussion}

Although enormous efforts have been made, the prognosis and overall survival of patients with NSCLC have not been improved yet. Considering NSCLC is a a highly 


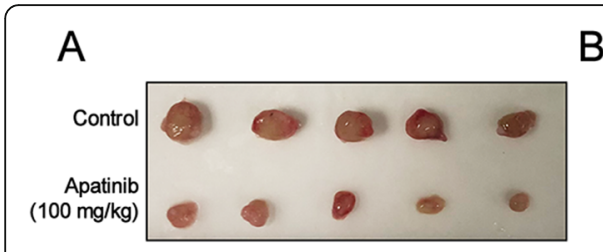

$\mathrm{F}$
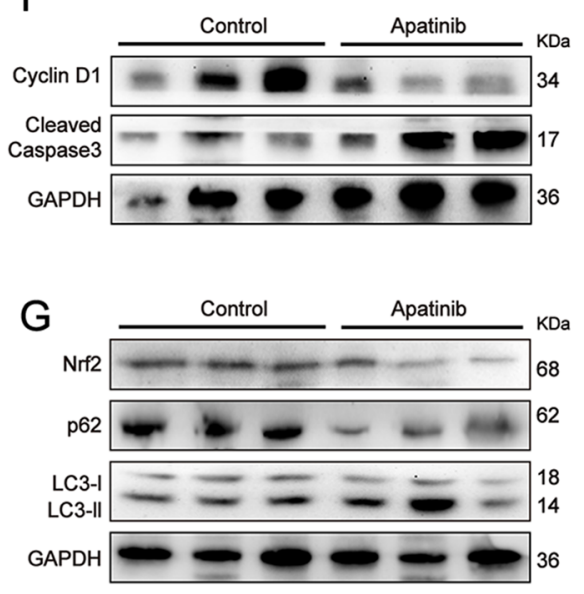
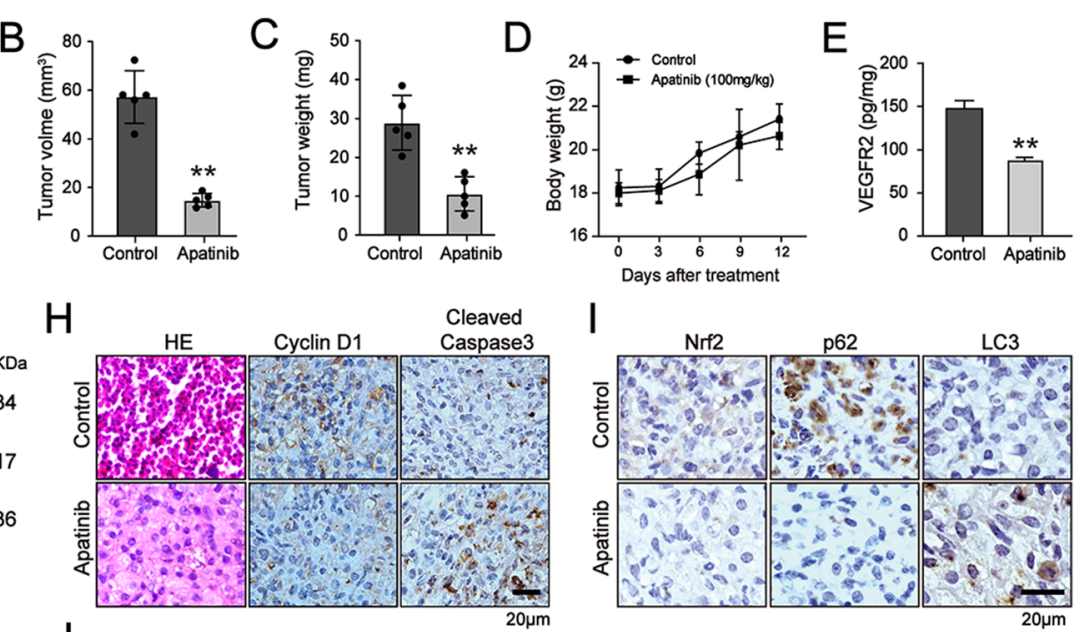

I

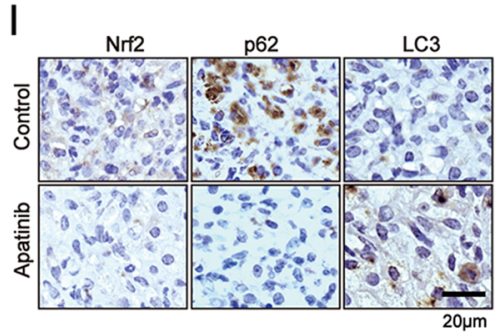

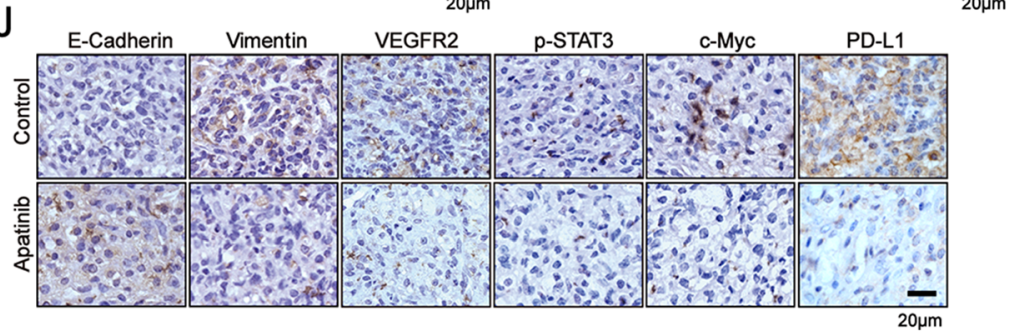

Fig. 7 Apatinib inhibited the growth of NSCLC cells in vivo. a Images of the xenograft tumors of the control and apatinib treatment group. $\mathbf{b}$ and c Tumor volume and tumor weight were measured. ${ }^{* *} p<0.01$ vs. control. The data are presented as mean \pm SD $(n=5)$. ${ }^{* *} p<0.01$ vs. control. $\mathbf{d}$ Body weights of mice were recorded every 3 days after indicated treatment. The data are presented as mean \pm SD $(n=5)$. e ELISA assay of VEGFR2 expression in tumors. The data are presented as mean \pm SD $(n=5) .{ }^{* *} p<0.01$ vs. control. $\mathbf{f}$ and $\mathbf{g}$ Western blot analysis of Cyclin D1, Cleaved Caspase 3, Nrf2, p62, and LC3 expressions in tumors. h-j H\&E staining of tumors and immunohistochemical staining of Cyclin D1, Cleaved Caspase 3, Nrf2, p62, LC3, E-cadherin, Vimentin, VEGFR2, P-STAT3, c-Myc, and PD-L1 expression in the tumors

vascularized tumor, angiogenesis blockade has been identified as an effective therapeutic strategy in inhibition of lung cancer progression. Apatinib is a highly selective TKI to VEGFR2 and has become an attractive drug in the treatment of NSCLC. However, the underlying mechanism of apatinib on NSCLC requires further illustration, which is critical for the clinical translational application of apatinib. A large number of studies have indicated that apatinib directly suppressed cell growth and triggered apoptosis in wide range of cancers [17, 25, 26, 31-33]. Consistent with the previous findings, we first observed the antitumor effects of apatinib on NSCL C A549 and H1299 cells in vitro and in vivo. Our results further showed that apatinib reduced the expression levels of phosphorylated STAT3 and its major downstream targets (c-Myc and PD-L1) in a concentrationand time-dependent manner. Meanwhile, apatinib treatment induced autophagic and apoptotic cell death by promoting ROS generation and inhibiting Nrf2 and p62 expression. Based on the multi-faceted evidence, we proposed a regulatory pathway of apatinib on proliferation inhibition and apoptosis induction in NSCLC (Fig. 8).

In the present study, we first observed that apatinib significantly inhibited cell viability and proliferation in
NSCLC cells in vitro. Our results further showed that apatinib suppressed the growth of A549 and AH299 cells by inducing G1 phase cell cycle arrest and apoptosis, which was also reported in PC9 gefitinib-resistant cancer cells [34]. Liu et al. reported that apatinib resensitized cisplatin-resistant A549 cells by increasing the levels of Cleaved Caspase 3, Cleaved Caspase 9 and Bax and decreasing Bcl-2 expression [35]. Our data also showed that apatinib had a similar impact on apoptosis in A549 and H1299 cells. Therefore, these results indicated that apatinib inhibited the growth of NSCLC cells through cell cycle arrest and apoptosis. Many studies have reported that A549 cells expressed a relative low level of VEGFR2 among NSCLC cell lines [36-38]. Our results presented evidence that NSCLC cells expressing low levels of VEGFR2 still responded to apatinib.

A previous report showed that apatinib suppressed the stemness of lung cancer stem cells in vivo by downregulating Hedgehog, Hippo, and Wnt pathways [39]. Our results also indicated that apatinib directly decreased the percentage of CD133-positive cells in A549 and H1299 cells culturing in a specific stem cell medium in vitro. It is acknowledged that CD133 is an important marker of lung cancer stem cells and NSCLC prognosis [40, 41]. 


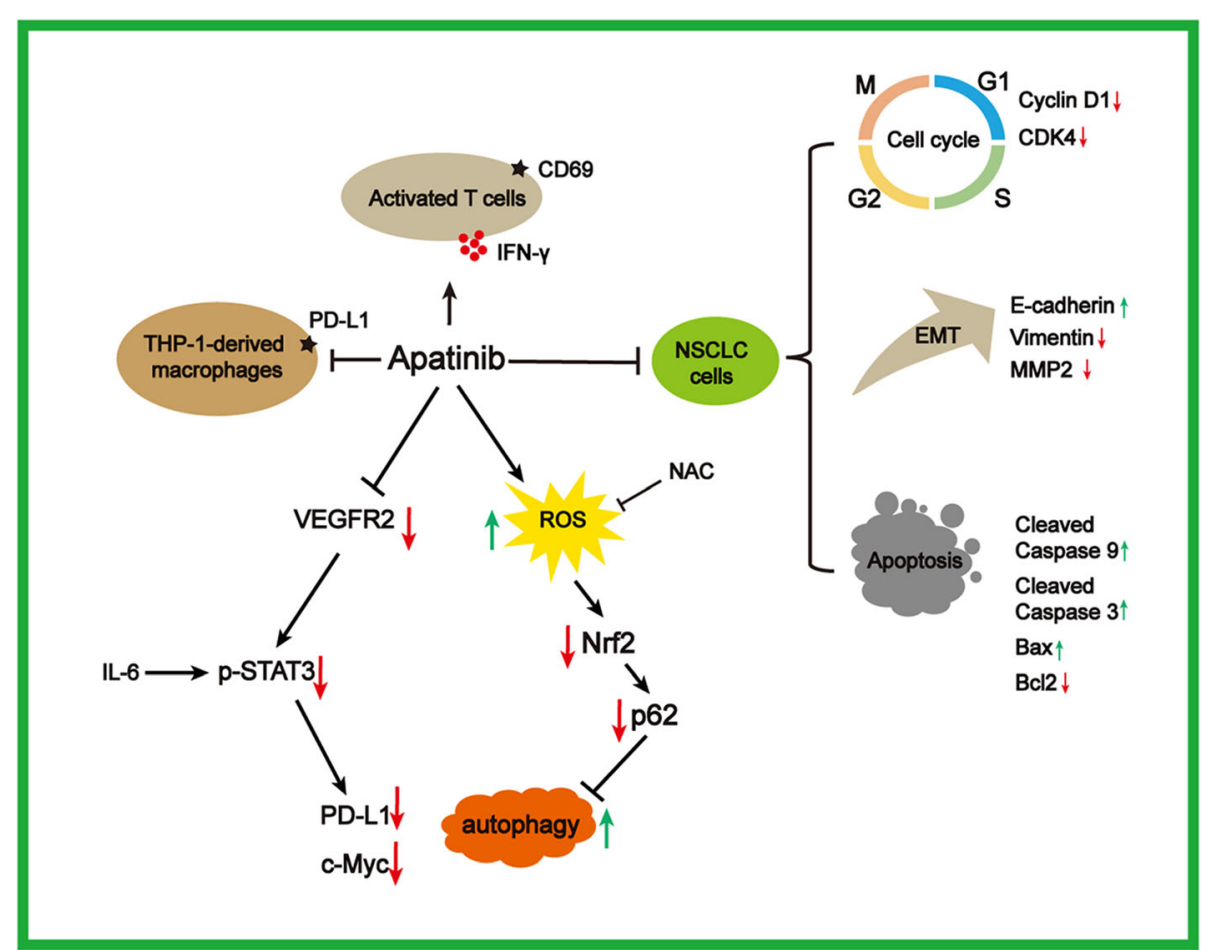

Fig. 8 Schematic diagram of how apatinib inhibited NSCLC. Apatinib suppressed cell proliferation, induced cell cycle arrest and apoptosis, and inhibited malignance of NSCLC cells. Mechanistically, apatinib downregulated PD-L1 and c-Myc expression through targeting VEGFR2/STAT3 pathway and induced ROS-triggered autophagy via decreasing Nrf2 and p62 in NSCLC cells. Apatinib further reduced immunosuppressive TME by suppressing PD-L1 expression in tumor-associated macrophages and partially restoring the activation of T cells

Simultaneously, numerous studies have demonstrated that apatinib inhibited migration and invasion in various tumors, including liver cancer [33], osteosarcoma [17], and gastric cancer [42]. Our study showed that apatinib markedly inhibited the migration and invasion capability of A549 and H1299 cells. Furthermore, we also showed that apatinib negatively regulated EMT-related markers, including E-cadherin, Vimentin and MMP2. Taken together, combined previous studies with our results, apatinib was considered as a promising therapeutic drug for NSCLC.

Recent studies have shown that the antitumor mechanisms of apatinib were associated with various multiple signaling pathways and specific regulatory molecules. In osteosarcoma, apatinib exerted its antitumor effects via VEGFR2/STAT3/BCL-2 pathway [26]. Apatinib inhibited osteosarcoma by targeting STAT3 and reducing PD-L1/PD-L2 [17, 43]. Furthermore, apatinib suppressed doxorubicin-induced stemness phenotype in osteosarcoma cells via STAT3 signaling [44]. In ovarian, liver and leukemia caner, apatinib regulated cell proliferation, apoptosis and EMT by inhibiting VEGFR2/STAT3 pathway $[31,32,45]$. Our results showed that apatinib $(5 \mu \mathrm{M}$ and $10 \mu \mathrm{M})$ induced a significant reduction of the total VEGFR2 level and had obvious cytotoxic effects in NSCLC cells. The cytotoxic effects of apatinib might lead to the reduction of total VEGFR2 in NSCLC cells. It is reported that multiple signaling pathways and molecules might be involved in the regulation of VEGFR2 expression [46-48]. Our previous study also indicated that apatinib suppressed lung cancer stem-like properties by inactivation of $\beta$-catenin signaling, which might further lead to the downregulation of VEGF and its receptor VEGFR2 [49]. STAT3 plays an essential role in tumor development and has been considered as a promising target for cancer treatment, especially in NSCLC therapy [50]. Meanwhile, STAT3 is constitutively hyper activated in EGFR-mutated NSCLC. In our study, we also found that apatinib decreased phosphorylated STAT3 in NSCL $\mathrm{C}$ in vitro and in vivo. $\mathrm{Li}$ et al. found that apatinib markedly potentiated the antitumor effect of gefitinib in NSCLC with T790M-related EGFR-TKI resistance [51]. Several studies demonstrated that apatinib plus EGFRTKIs (gefitinib) exhibited a tolerable safety profile and encouraging antitumor activity for advanced EGFRmutant NSCLC [52-54]. Thus, the efficacy of apatinib in EGFR-mutant NSCLC might be related with the inactivation of STAT3. Our results suggest that apatinib may be an alternative therapeutic approach for patients with advance lung cancer.

We further observed that PD-L1 expression was also decreased in apatinib-treated NSCLC cells. PD-L1, one 
of the hot spots in current immunotherapy, can be induced by the intrinsic oncogenic pathways, including STAT3 signaling [55]. Overexpression of PD-L1 was contributed to the poor prognosis of lung cancer [56]. A recent study illustrated that low-dose apatinib with antiPD-L1 antibody optimized tumor microenvironment by alleviating hypoxia, promoting the infiltration of CD8 (+) T cells, reducing recruitment of tumor-associated macrophages in lung cancer [15]. STAT3 pathway was reported to be the most critical downstream of IL-6 signaling that modulated PD-L1 in lung cancer cells. It has been reported that IL- 6 regulated inflammation and the immune response by promoting the phosphorylation of STAT3 [57]. We noted that apatinib not only inhibited IL-6-mediated upregulation of phosphorylated STAT3 and PD-L1 in NSCLC cells, but also suppressed PD-L1 activation in THP-1- derived macrophages which were stimulated by CM from A549 and H1299 cells. We also found that pretreatment of apatinib partially restored the activation of Jurket T cells co-cultured with NSCLC cells, as evidenced by the increase of CD69 expression and IFN- $\gamma$ secretion. Therefore, our results indicated that apatinib might exert its inhibitory effects on NSCL C by targeting VEGFR2/STAT3/PD-L1 signaling in both NSCLC cells and tumor derived macrophages and partially restoring theactivation of $\mathrm{T}$ cells. Collectively, our data suggested that apatinib might reduce the immunosuppressive TME in NSCLC.

Traditional chemotherapeutic drugs used in cancer therapy generally induced ROS production and disturbed the intracellular reduction capacity, resulting in the induction of cell death [58]. Apatinib has been reported to promote ROS-dependent apoptosis in many cancer cells, such as ovarian cancer cells [24] and breast cancer cells [59]. Our results showed that apatinib induced ROS production in NSCLC A549 and H1299 cells. Our previous study found that apatinib-triggered ROS induced mitochondrial transmembrane potential reduction, leading to the mitochondrial dysfunction [49]. VEGFR2-downstream signaling is linked to mitochondria biology in cancer [60,61], which might be involved in apatinib-triggered ROS. It is reported that the transcriptional factor Nrf2 regulates the expression of antioxidant genes and plays a vital role in redox homeostasis. Emerging evidence has shown that Nrf2 pathway is frequently dysregulated in lung cancer and overactivation of the pathway is associated with poor prognosis of NSCLC $[62,63]$. Our results indicated that apatinib inhibited Nrf2 expression in NSCLC in a concentration- and time-dependent manner. Consistent with our results, Sun et al. found that apatinib downregulated Nrf2 and promoted ROS production in ovarian cancer [24]. Therefore, we speculated that apatinib promoted ROS generation to inhibit NSCLC by targeting
Nrf2 and ROS exerted a critical role in apatinib-induced apoptosis in NSCLC.

Of note, autophagy is very important in regulating cell survival and homeostasis. It has been demonstrated that autophagy and apoptosis interact with each other to induce cell death. Previous studies showed apatinib promoted autophagy and apoptosis in many tumor types [26]. However, litter is known about the role of apatinib on autophagy in NSCLC. In our present study, we showed that apatinib significantly enhanced autophagic and apoptotic cell death in NSCLC in vitro and in vivo. It has been demonstrated that autophagy and the Nrf2 system are the major cellular defense mechanisms against oxidative stress. These two systems affect each other via p62 (autophagy receptor protein) and Keap1 (the Nrf2 substrate adaptor for the Cul3 E3 ubiquitin ligase) [64]. Our results indicated that pretreatment of antioxidant NAC reversed apatinib-downregulated Nrf2 and -upregulated autophagy. We further showed that the effects of apatinib on cell proliferation and apoptosis were attenuated by NAC. Therefore, these data indicated that apatinib induced ROS production and reduced Nrf2 and p62 expression, which further led to the autophagy and apoptosis of NSCLC cells.

\section{Conclusion}

In conclusion, our results showed that apatinib suppressed proliferation and promoted autophagic and apoptotic cell death of NSCLC by regulating both VEGF R2/STAT3/PD-L1/c-Myc and ROS/Nrf2/p62 signaling. Findings from the present study indicated that apatinib might be a promising therapeutic agent for NSCLC.

\section{Abbreviations}

NSCLC: Non-small cell lung cancer; VEGF: Vascular endothelial growth factor; VEGFR: Vascular endothelial growth factor receptor; TKI: Tyrosine kinase inhibitor; TME: Tumor microenvironment; PD-1: Programmed death 1; PD L1: Programmed death ligand 1; STAT: Signal transducer and activator of transcription; Nrf2: Nuclear factor erythroid-derived 2-like 2; ROS: Reactive oxygen species; Keap1: Kelch-like ECH-associated protein 1; NAC: N-acetyl-Lcysteine; CM: Conditioned-medium; IFNy: Interferon-ү; EMT: Epithelialmesenchymal transition; IL-6: Interleukin-6

\section{Supplementary Information}

The online version contains supplementary material available at https://doi. org/10.1186/s13046-021-02069-4.

\section{Additional file 1: Supplementary. Raw data for the blots.}

\section{Acknowledgements}

Not applicable.

\section{Authors' contributions}

$J Z$ and $C X$ designed and performed experiments, and analyzed the data. CX, $X L, X Z, C L, M G, Y C$, and JY performed experiments. JZ and CX wrote the manuscript draft. CZ and JZ supervised the study, interpreted the data and provided funding for this study. CZ had the primary responsibility for the study. All authors read and approved the final manuscript. 


\section{Funding}

This work was supported by grants from the National Natural Science Foundation of China (No. 81803210 and No. 81973026), the Natural Science Foundation of Jiangsu Province (BK20180217), the China Postdoctoral Science Foundation Funded Project (2018M642280 and 2020T130312), Suzhou Talent Training Program (GSWS2020075), and the Research Project of Nanjing Medical University (No. 2018KF0096).

\section{Availability of data and materials}

The datasets supporting the conclusions of this article are included within the article.

\section{Declarations}

\section{Ethics approval and consent to participate}

Mice were handled under the recommendations in the guidelines of the Animal Care and Welfare Committee of Nanjing Medical University (IACUC1907001).

\section{Consent for publication}

All contributing authors agreed to the publication of this article.

\section{Competing interests}

The authors declared that they had no competing interests.

\section{Author details}

${ }^{1}$ Department of Nutrition and Food Safety, School of Public Health, Nanjing Medical University, 101 Longmian Ave, Jiangning, Nanjing 211166, China. ${ }^{2}$ Department of Laboratory, The Affiliated Suzhou Hospital of Nanjing Medical University, Suzhou Municipal Hospital, Gusu School, Nanjing Medical University, 242 Guangji Rd, Suzhou 215008, China. ${ }^{3}$ Cancer Research Division, Center for Global Health, School of Public Health, Nanjing Medical University, Nanjing 211166, Jiangsu, China.

\section{Received: 6 April 2021 Accepted: 10 August 2021}

\section{Published online: 24 August 2021}

\section{References}

1. Chen W, Zheng R, Baade PD, Zhang S, Zeng H, Bray F, et al. Cancer statistics in China, 2015. CA Cancer J Clin. 2016;66(2):115-32. https://doi.org/10.3322/ caac. 21338.

2. Siegel RL, Miller KD, Jemal A. Cancer statistics, 2020. CA Cancer J Clin. 2020; 70(1):7-30. https://doi.org/10.3322/caac.21590.

3. Molina JR, Yang P, Cassivi SD, Schild SE, Adjei AA. Non-small cell lung cancer: epidemiology, risk factors, treatment, and survivorship. Mayo Clin Proc. 2008;83(5):584-94. https://doi.org/10.1016/S0025-6196(11)60735-0.

4. Zappa C, Mousa SA. Non-small cell lung cancer: current treatment and future advances. Transl Lung Cancer Res. 2016;5(3):288-300. https://doi. org/10.21037/tlcr.2016.06.07.

5. Zhang C, Leighl NB, Wu YL, Zhong WZ. Emerging therapies for non-small cell lung cancer. J Hematol Oncol. 2019;12(1):45. https://doi.org/10.1186/s13 045-019-0731-8.

6. Yuan M, Huang LL, Chen JH, Wu J, Xu Q. The emerging treatment landscape of targeted therapy in non-small-cell lung cancer. Signal Transduct Target Ther. 2019;4(1):61. https://doi.org/10.1038/s41392-0190099-9.

7. Daum S, Hagen $H$, Naismith E, Wolf D, Pircher A. The role of antiangiogenesis in the treatment landscape of non-small cell lung cancer new combinational approaches and strategies of neovessel inhibition. Front Cell Dev Biol. 2020;8:610903.

8. Fontanella C, Ongaro E, Bolzonello S, Guardascione M, Fasola G, Aprile G. Clinical advances in the development of novel VEGFR2 inhibitors. Ann Translat Med. 2014;2(12):123. https://doi.org/10.3978/j.issn.2305-5839.2014. 08.14 .

9. Zhang $\mathrm{H}$. Apatinib for molecular targeted therapy in tumor. Drug Des Devel Ther. 2015;9:6075-81.

10. Fathi Maroufi N, Rashidi MR, Vahedian V, Akbarzadeh M, Fattahi A, Nouri M. Therapeutic potentials of Apatinib in cancer treatment: possible mechanisms and clinical relevance. Life Sci. 2020;241:117106. https://doi. org/10.1016/j.lfs.2019.117106.
11. Liang J, Gu W, Jin J, Zhang H, Chen Z, Tang Y, et al. Efficacy and safety of apatinib as third- or further-line therapy for patients with advanced NSCLC: a retrospective study. Therapeut Adv Med Oncol. 2020;12: 1758835920968472.

12. Zhou C, Wang Y, Zhao J, Chen G, Liu Z, Gu K, et al. Efficacy and biomarker analysis of Camrelizumab in combination with Apatinib in patients with advanced nonsquamous NSCLC previously treated with chemotherapy. Clin Cancer Res. 2021;27(5):1296-304.

13. $X u$ Y, Huang Z, Lu H, Yu X, Li Y, Li W, et al. Apatinib in patients with extensive-stage small-cell lung cancer after second-line or third-line chemotherapy: a phase II, single-arm, multicentre, prospective study. Br J Cancer. 2019;121(8):640-6. https://doi.org/10.1038/s41416-019-0583-6.

14. Liang P, Ballou B, Lv X, Si W, Bruchez MP, Huang W, et al. Monotherapy and combination therapy using anti-angiogenic nanoagents to fight cancer. Adv Mater. 2021;33(15):e2005155.

15. Zhao S, Ren S, Jiang T, Zhu B, Li X, Zhao C, et al. Low-dose Apatinib optimizes tumor microenvironment and potentiates antitumor effect of PD1/PD-L1 blockade in lung cancer. Cancer Immunol Res. 2019;7(4):630-43. https://doi.org/10.1158/2326-6066.CIR-17-0640.

16. Wang Q, Gao J, Di W, Wu X. Anti-angiogenesis therapy overcomes the innate resistance to PD-1/PD-L1 blockade in VEGFA-overexpressed mouse tumor models. Cancer Immunol Immunother. 2020;69(9):1781-99. https:// doi.org/10.1007/s00262-020-02576-X.

17. Zheng B, Ren T, Huang Y, Guo W. Apatinib inhibits migration and invasion as well as PD-L1 expression in osteosarcoma by targeting STAT3. Biochem Biophys Res Commun. 2018;495(2):1695-701. https://doi.org/10.1016/j.bbrc.2 017.12.032.

18. Cai X, Wei B, Li L, Chen X, Liu W, Cui J, et al. Apatinib enhanced anti-PD-1 therapy for colon cancer in mice via promoting PD-L1 expression. Int Immunopharmacol. 2020;88:106858. https://doi.org/10.1016/j.intimp.2020.1 06858.

19. Yang $Y$, Wang $C$, Sun $H$, Jiang Z, Zhang Y, Pan Z. Apatinib prevents natural killer cell dysfunction to enhance the efficacy of anti-PD-1 immunotherapy in hepatocellular carcinoma. Cancer Gene Ther. 2021;28(1-2):89-97. https:// doi.org/10.1038/s41417-020-0186-7.

20. Liu Y, Lang F, Yang C. NRF2 in human neoplasm: Cancer biology and potential therapeutic target. Pharmacol Ther. 2021;217:107664. https://doi. org/10.1016/j.pharmthera.2020.107664.

21. Komatsu M, Kurokawa H, Waguri S, Taguchi K, Kobayashi A, Ichimura Y, et al The selective autophagy substrate p62 activates the stress responsive transcription factor Nrf2 through inactivation of Keap1. Nat Cell Biol. 2010; 12(3):213-23. https://doi.org/10.1038/ncb2021.

22. Mathew R, Karp CM, Beaudoin B, Vuong N, Chen G, Chen HY, et al. Autophagy suppresses tumorigenesis through elimination of p62. Cell. 2009; 137(6):1062-75. https://doi.org/10.1016/j.cell.2009.03.048.

23. Ichimura Y, Komatsu M. Activation of p62/SQSTM1-Keap1-nuclear factor erythroid 2-related factor 2 pathway in cancer. Front Oncol. 2018;8:210. https://doi.org/10.3389/fonc.2018.00210.

24. Sun X, Li J, Li Y, Wang S, Li Q. Apatinib, a novel tyrosine kinase inhibitor, promotes ROS-dependent apoptosis and autophagy via the $\mathrm{Nrf} / \mathrm{HO}-1$ pathway in ovarian cancer cells. Oxidative Med Cell Longev. 2020;2020: 3145182.

25. Feng $H$, Cheng $X$, Kuang J, Chen L, Yuen S, Shi M, et al. Apatinib-induced protective autophagy and apoptosis through the AKT-mTOR pathway in anaplastic thyroid cancer. Cell Death Dis. 2018;9(10):1030. https://doi.org/1 0.1038/s41419-018-1054-3.

26. Liu K, Ren T, Huang Y, Sun K, Bao X, Wang S, et al. Apatinib promotes autophagy and apoptosis through VEGFR2/STAT3/BCL-2 signaling in osteosarcoma. Cell Death Dis. 2017:8(8):e3015. https://doi.org/10.1038/ cddis.2017.422.

27. Zhu J, Wang S, Chen Y, Li X, Jiang Y, Yang X, et al. miR-19 targeting of GSK3beta mediates sulforaphane suppression of lung cancer stem cells. J Nutr Biochem. 2017:44:80-91. https://doi.org/10.1016/j.jnutbio.2017.02.020.

28. Tirino V, Camerlingo R, Franco R, Malanga D, La Rocca A, Viglietto G, et al. The role of CD133 in the identification and characterisation of tumourinitiating cells in non-small-cell lung cancer. Eur J Cardio-Thorac Surg. 2009; 36(3):446-53. https://doi.org/10.1016/j.ejcts.2009.03.063.

29. Bertolini G, Roz L, Perego P, Tortoreto M, Fontanella E, Gatti L, et al. Highly tumorigenic lung cancer CD133+ cells display stem-like features and are spared by cisplatin treatment. Proc Natl Acad Sci U S A. 2009;106(38):162816. https://doi.org/10.1073/pnas.0905653106. 
30. Kim S, Jang JY, Koh J, Kwon D, Kim YA, Paeng JC, et al. Programmed cell death ligand-1-mediated enhancement of hexokinase 2 expression is inversely related to T-cell effector gene expression in non-small-cell lung cancer. J Exp Clin Cancer Res. 2019;38(1):462. https://doi.org/10.1186/s13046-019-1407-5.

31. Ding J, Cheng XY, Liu S, Ji HY, Lin M, Ma R, et al. Apatinib exerts antitumour effects on ovarian cancer cells. Gynecol Oncol. 2019;153(1):165-74. https://doi.org/10.1016/j.ygyno.2019.01.010.

32. Deng M, Zha J, Zhao H, Jia X, Shi Y, Li Z, et al. Apatinib exhibits cytotoxicity toward leukemia cells by targeting VEGFR2-mediated prosurvival signaling and angiogenesis. Exp Cell Res. 2020;390(1):111934. https://doi.org/10.1016/j. yexcr.2020.111934

33. He X, Huang Z, Liu P, Li Q, Wang M, Qiu M, et al. Apatinib inhibits the invasion and metastasis of liver cancer cells by downregulating MMPrelated proteins via regulation of the NF-kappaB signaling pathway. Biomed Res Int. 2020;2020:3126182

34. Song YA, Ma T, Zhang XY, Cheng XS, Olajuyin AM, Sun ZF, et al. Apatinib preferentially inhibits PC9 gefitinib-resistant cancer cells by inducing cell cycle arrest and inhibiting VEGFR signaling pathway. Cancer Cell Int. 2019; 19(1):117. https://doi.org/10.1186/s12935-019-0836-8.

35. Liu ZL, Jin BJ, Cheng CG, Zhang FX, Wang SW, Wang Y, et al. Apatinib resensitizes cisplatin-resistant non-small cell lung carcinoma A549 cell through reversing multidrug resistance and suppressing ERK signaling pathway. Eur Rev Med Pharmacol Sci. 2017;21(23):5370-7. https://doi.org/1 0.26355/eurrev_201712_13922

36. Hu C, Zhu P, Xia Y, Hui K, Wang M, Jiang X. Role of the NRP-1-mediated VEGFR2-independent pathway on radiation sensitivity of non-small cell lung cancer cells. J Cancer Res Clin Oncol. 2018;144(7):1329-37. https://doi.org/1 0.1007/s00432-018-2667-8.

37. Watanabe H, Ichihara E, Kayatani H, Makimoto G, Ninomiya K, Nishii K, et al. VEGFR2 blockade augments the effects of tyrosine kinase inhibitors by inhibiting angiogenesis and oncogenic signaling in oncogene-driven nonsmall-cell lung cancers. Cancer Sci. 2021;112(5):1853-64. https://doi.org/1 $0.1111 /$ cas.14801.

38. Liu L, Qiao Y, Hu C, Liu Y, Xia Y, Wang L, et al. Endostatin exerts radiosensitizing effect in non-small cell lung cancer cells by inhibiting VEGF R2 expression. Clin Transl Oncol. 2016;18(1):18-26. https://doi.org/10.1007/ s12094-015-1319-6.

39. Yang B, Wang Y, Chen Z, Feng YM, Shi LL. Effects of Apatinib on the "Stemness" of non-small-cell lung cancer cells in vivo and its related mechanisms. Can Respir J. 2020;2020:2479369.

40. Mizugaki H, Sakakibara-Konishi J, Kikuchi J, Moriya J, Hatanaka KC, Kikuchi E, et al. CD133 expression: a potential prognostic marker for non-small cell lung cancers. Int J Clin Oncol. 2014;19(2):254-9. https://doi.org/10.1007/s1 0147-013-0541-X.

41. Zhu JY, Yang $X$, Chen $Y$, Jiang Y, Wang SJ, Li Y, et al. Curcumin suppresses lung cancer stem cells via inhibiting Wnt/beta-catenin and sonic hedgehog pathways. Phytother Res. 2017;31(4):680-8. https://doi.org/10.1002/ptr.5791.

42. Zhou N, Zhang C, Liu D, Liu K, Wang G, Zhu H, et al. Apatinib in combination with S-1 as first-line treatment in patients with advanced metastatic gastric cancer: results from an open, exploratory, single-arm, phase ii trial. Oncologist. 2021;26(3):e374-e81. https://doi.org/10.1002/ onco.13613.

43. Zheng B, Zhou C, Qu G, Ren C, Yan P, Guo W, et al. VEGFR2 promotes metastasis and PD-L2 expression of human osteosarcoma cells by activating the STAT3 and RhoA-ROCK-LIMK2 pathways. Front Oncol. 2020;10:543562. https://doi.org/10.3389/fonc.2020.543562.

44. Tian ZC, Wang JQ, Ge H. Apatinib ameliorates doxorubicin-induced migration and cancer stemness of osteosarcoma cells by inhibiting Sox2 via STAT3 signalling. J Orthop Translat. 2020;22:132-41. https://doi.org/10.1016/j. jot.2019.07.003.

45. Wen S, Shao G, Zheng J, Zeng H, Luo J, Gu D. Apatinib regulates the cell proliferation and apoptosis of liver cancer by regulation of VEGFR2/STAT3 signaling. Pathol Res Pract. 2019;215(4):816-21. https://doi.org/10.1016/j. prp.2019.01.021.

46. Easwaran V, Lee SH, Inge L, Guo L, Goldbeck C, Garrett E, et al. beta-catenin regulates vascular endothelial growth factor expression in colon cancer. Cancer Res. 2003;63(12):3145-53.

47. Hegde M, Guruprasad KP, Ramachandra L, Satyamoorthy K, Joshi MB. Interleukin-6-mediated epigenetic control of the VEGFR2 gene induces disorganized angiogenesis in human breast tumors. J Biol Chem. 2020; 295(34):12086-98. https://doi.org/10.1074/jbc.RA120.012590.
48. Yang Y, Xia L, Wu Y, Zhou H, Chen X, Li H, et al. Programmed death ligand1 regulates angiogenesis and metastasis by participating in the c-JUN/ VEGFR2 signaling axis in ovarian cancer. Cancer Commun. 2021;41(6):51127. https://doi.org/10.1002/cac2.12157.

49. Zhu J, Li X, Liang C, Zhou X, Ge M, Chen Y, et al. Apatinib suppresses lung cancer stem-like cells by complex interplay between beta-catenin signaling and mitochondrial ROS accumulation. Cell Death Discov. 2021;7(1):102. https://doi.org/10.1038/s41420-021-00480-6.

50. Kim BH, Yi EH, Ye SK. Signal transducer and activator of transcription 3 as a therapeutic target for cancer and the tumor microenvironment. Arch Pharm Res. 2016;39(8):1085-99. https://doi.org/10.1007/s12272-016-0795-8.

51. Li F, Zhu T, Cao B, Wang J, Liang L. Apatinib enhances antitumour activity of EGFR-TKIs in non-small cell lung cancer with EGFR-TKI resistance. Eur J Cancer. 2017:84:184-92. https://doi.org/10.1016/j.ejca.2017.07.037.

52. Zhang Z, Zhang Y, Luo F, Ma Y, Fang W, Zhan J, et al. Dual blockade of EGFR and VEGFR pathways: results from a pilot study evaluating apatinib plus gefitinib as a first-line treatment for advanced EGFR-mutant non-small cell lung cancer. Clin Translat Med. 2020;10(2):e33. https://doi.org/10.1002/ctm2.33.

53. Zhao H, Yao W, Min X, Gu K, Yu G, Zhang Z, et al. Apatinib plus gefitinib as first-line treatment in advanced EGFR-mutant NSCLC: the phase III ACTIVE study (CTONG1706). J Thorac Oncol. 2021;\$1556-0864(21)02177-8. https:// doi.org/10.1016/j.jtho.2021.05.006.

54. Zhang Z, Luo F, Zhang Y, Ma Y, Hong S, Yang Y, et al. The ACTIVE study protocol: apatinib or placebo plus gefitinib as first-line treatment for patients with EGFR-mutant advanced non-small cell lung cancer (CTONG1706). Cancer Commun. 2019;39(1):69. https://doi.org/10.1186/s4 0880-019-0414-4.

55. Chen J, Jiang CC, Jin L, Zhang XD. Regulation of PD-L1: a novel role of prosurvival signalling in cancer. Ann Oncol. 2016;27(3):409-16. https://doi.org/1 0.1093/annonc/mdv615

56. Zhou ZJ, Zhan P, Song Y. PD-L1 over-expression and survival in patients with non-small cell lung cancer: a meta-analysis. Transl Lung Cancer Res. 2015;4(2):203-8. https://doi.org/10.3978/j.issn.2218-6751.2015.03.02.

57. Mohrherr J, Uras IZ, Moll HP, Casanova E. STAT3: versatile functions in nonsmall cell lung cancer. Cancers. 2020;12(5):1107.

58. Teppo HR, Soini Y, Karihtala P. Reactive oxygen species-mediated mechanisms of action of targeted cancer therapy. Oxidative Med Cell Longev. 2017;2017:1485283.

59. Tang D, Ma J, Chu Z, Wang X, Zhao W, Zhang Q. Apatinib-induced NFkappaB inactivation sensitizes triple-negative breast cancer cells to doxorubicin. Am J Transl Res. 2020;12(7):3741-53.

60. Nobrega-Pereira S, Caiado F, Carvalho T, Matias I, Graca G, Goncalves LG, et al. VEGFR2-mediated reprogramming of mitochondrial metabolism regulates the sensitivity of acute myeloid leukemia to chemotherapy. Cancer Res. 2018;78(3):731-41. https://doi.org/10.1158/0008-5472.CAN-17-11 66.

61. Grillo E, Corsini M, Ravelli C, Zammataro L, Bacci M, Morandi A, et al. Expression of activated VEGFR2 by R1051Q mutation alters the energy metabolism of Sk-Mel-31 melanoma cells by increasing glutamine dependence. Cancer Lett. 2021;507:80-8. https://doi.org/10.1016/j.canlet.2 021.03.007.

62. Zhao J, Lin X, Meng D, Zeng L, Zhuang R, Huang S, et al. Nrf2 mediates metabolic reprogramming in non-small cell lung cancer. Front Oncol. 2020; 10:578315. https://doi.org/10.3389/fonc.2020.578315.

63. Hammad A, Namani A, Elshaer M, Wang XJ, Tang X. "NRF2 addiction" in lung cancer cells and its impact on cancer therapy. Cancer Lett. 2019;467: 40-9. https://doi.org/10.1016/j.canlet.2019.09.016.

64. Jiang $T$, Harder B, Rojo de la Vega M, Wong PK, Chapman E, Zhang DD. p62 links autophagy and Nrf2 signaling. Free Radic Biol Med. 2015;88(Pt B):199_ 204.

\section{Publisher's Note}

Springer Nature remains neutral with regard to jurisdictional claims in published maps and institutional affiliations. 